\title{
Pathways to relativistic curved momentum spaces: de Sitter case study
}

\author{
Giovanni AMELINO-CAMELIA, ${ }^{1,2}$ Giulia GUBITOSI, ${ }^{1,2}$ and Giovanni PALMISANO ${ }^{1,2}$ \\ ${ }^{1}$ Dipartimento di Fisica, Università di Roma "La Sapienza", P.le A. Moro 2, 00185 Roma, Italy \\ ${ }^{2}$ INFN, Sez. Roma1, P.le A. Moro 2, 00185 Roma, Italy
}

\begin{abstract}
Several arguments suggest that the Planck scale could be the characteristic scale of curvature of momentum space. As other recent studies we assume that the metric of momentum space determines the condition of on-shellness while the momentum-space affine connection governs the form of the law of composition of momenta. We show that the possible choices of laws of composition of momenta are more numerous than the possible choices of affine connection on a momentum space. This motivates us to propose a new prescription for associating an affine connection to momentum composition, which we compare to the one most used in the recent literature. We find that the two prescriptions lead to the same picture of the so-called $\kappa$-momentum space, with de Sitter metric and $\kappa$-Poincaré connection. We also examine in greater detail than ever before the DSR-relativistic properties of $\kappa$-momentum space, particularly in relation to its noncommutative law of composition of momenta. We then show that in the case of "proper de Sitter momentum space", with the de Sitter metric and its Levi-Civita connection, the two prescriptions are inequivalent. Our novel prescription leads to a picture of proper de Sitter momentum space which is DSR-relativistic and is characterized by a commutative law of composition of momenta, a possibility for which no explicit curved-momentum-space picture had been previously found. We argue that our construction provides a natural test case for the study of momentum spaces with commutative, and yet deformed, laws of composition of momenta. Moreover, it can serve as laboratory for the exploration of the properties of DSR-relativistic theories which are not connected to group-manifold momentum spaces and Hopf algebras.
\end{abstract}

\section{CONTENTS}

I. Introduction

II. Geometry of momentum space from on-shell relation and momentum conservation 3

A. Geometrical Interpretation of the on-shell relation 4

B. Standard geometrical interpretation of the Composition Law 4

C. A novel geometrical interpretation of the Composition Law

D. Aside on an ambiguity in the link from composition law to affine connection 6

III. Differences between the standard and our novel prescription for the geometry of momentum space

IV. Two $\kappa$-de Sitter momentum spaces 11

A. $\kappa$-dS composition law from $\kappa$-connection $\quad[12$

B. Two $\kappa$-dS geometries

\begin{tabular}{lr} 
V. "Proper" de Sitter momentum space & 14 \\
\hline
\end{tabular}

A. Weakly-proper dS 14

B. Proper dS

\begin{tabular}{lr} 
VI. DSR-relativistic properties of proper-dS and $\kappa$-dS momentum spaces & 16 \\
\hline
\end{tabular}

A. The starting point of the relativistic properties of Minkowski momentum space 17

B. DSR-relativistic compatibility of "proper dS" momentum space

\begin{tabular}{ll} 
C. DSR compatibility of weakly-proper de Sitter & 20 \\
\hline
\end{tabular}

D. Aside on more general forms of DSR-relativistic compatibility 20

E. DSR-relativistic compatibility of $\kappa$-dS momentum space $\quad 22$

VII. Relativistic consistency as a geometric property 24

A. Diffeomorphisms of proper-dS momentum space

B. All-order DSR-relativistic compatibility of proper-dS momentum space 27 


\section{INTRODUCTION}

Max Born argued in 1938 [1], inspired by Born duality, that curvature of momentum space might be a needed step toward quantum gravity. For several decades this proposal attracted little or no interest (see, however, Ref. 2]), but over the last decade several independent arguments pointed more or less explicitly toward a role for the Planck scale in characterizing a non-trivial geometry of momentum space (see, e.g., Refs. 3 10]). Among the reasons of interest in this possibility we should mention approaches to the study of the quantum-gravity problem based on spacetime noncommutativity, particularly when considering models with "Lie-algebra spacetime noncommutativity", $\left[x_{\mu}, x_{\nu}\right]=i \zeta_{\mu \nu}^{\sigma} x_{\sigma}$, where the momentum space on which spacetime coordinates generate translations is evidently curved (see, e.g., Ref [1]). Also in the Loop Quantum Gravity approach [12] one can adopt a perspective suggesting momentum-space curvature (see, e.g., Ref [13]). And one should take notice of the fact that the only quantum gravity we actually know how to solve, quantum gravity in the $2+1$-dimensional case, definitely does predict a curved momentum space (see, e.g., Refs. [14 17]).

We here focus on the perspective on Planck-scale-curved momentum spaces adopted in the recently proposed "relative locality framework" 9], which essentially abstracts the insight gained in the study of $2+1 \mathrm{D}$ quantum gravity, providing a picture for how the geometry of momentum space can play a role in describing Planck-scale-deformed relativistic kinematics. This proposal links the metric on momentum space to the form of the on-shell/dispersion relation, while the affine connection on momentum space is linked to the form of the law of composition of momenta, which in turn determines the energy-momentum conservation laws.

One of the issues that is most relevant for the analysis we here report concerns [18, 19] the identification of the requirements that must be enforced on the geometry of momentum space in order to allow the formulation of relativistic theories. Since special-relativistic laws of transformation cannot be symmetries of any curved momentum space, relativistic invariance must be inevitably implemented according to the proposal of "DSR relativistic theories" [4] (also see Refs. [20 25]), theories with two relativistic invariants, the speed-of-light scale $c$ and a length/inversemomentum scale: the scale that characterizes the geometry of momentum space must in fact be an invariant if the theories on such momentum spaces are to be relativistic. Several grey areas however remain toward the understanding of the compatibility between metric and affine connection on momentum space that must be enforced in order to have a relativistic picture.

The other aspect which is of strong interest to us is the link between affine connection on momentum space and law of composition of momenta. We here show that the possible choices of laws of composition of momenta are more numerous than the possible choices of affine connection on a momentum space, an issue which was not noticed in the previous related literature and which we feel should play an important role in future work in this research area. Partly inspired by this observation, we here propose a new prescription for associating an affine connection to momentum composition, which we compare to the one most used in the recent literature. As arena for comparing the two prescriptions we focus on the case of momentum spaces with de Sitter metric. We find that the two prescriptions lead to the same picture of the so-called $\kappa$-momentum space, with de Sitter metric and $\kappa$-Poincaré connection. We also examine in greater detail than ever before the DSR-relativistic properties of $\kappa$-momentum space, particularly in relation to its noncommutative law of composition of momenta. We then show that in the case of "proper de Sitter momentum space", with the de Sitter metric and its Levi-Civita connection, the two prescriptions are inequivalent. Our novel prescription leads to a picture of proper de Sitter momentum space which is DSR-relativistic and is characterized by a commutative law of composition of momenta.

As it will become clearer as we go along, one of the elements of interest that motivated our analysis is the search of a natural candidate of momentum space with a commutative composition law. All the momentum spaces that have attracted attention so far have noncommutative composition law, but it is an interesting hypothesis for physics the one in which the composition law is indeed deformed but still preserves the property of commutativity. We argue 
that our construction of a "proper de Sitter momentum space" provides the first natural example of momentum space with commutative, and yet deformed, law of composition of momenta.

In order to keep our presentation clear and compact we focus on the 1+1-dimensional case, where all the key conceptual challenges are already present but formulas are more compact and derivations are less tedious.

The next section sets up the analysis by reviewing the previously most studied prescription and characterizing our novel prescription for associating a geometry of momentum space to on-shellness and momentum composition.

In Section III we discuss the differences between these two prescriptions and we establish the fact that the possible choices of laws of composition of momenta are more numerous than the possible choices of affine connection on a momentum space.

Then in Section IV we re-derive the structure of momentum space which can be inspired by the structure of the $\kappa$-Poincare Hopf algebra. This leads to the already much studied $\kappa$-momentum space, with de Sitter metric and a torsionful affine connection. We also show that actually the prescriptions so far given in formulating the geometry of momentum space within the relative-locality framework are affected by an ambiguity. This ambiguity is merely academic since the alternative geometries allowed by it give rise to equivalent relativistic kinematics, but for momentum spaces with torsion it weakens the link from the observables of the theory to the geometry of momentum space. We discuss how, exploiting this ambiguity, one can find two alternative formulations of the $\kappa$-momentum space, both with the same momentum space metric and with the same physical predictions, but formulated in terms of different affine connections.

Section V proposes our new "proper de Sitter momentum space", obtained, in the sense of our novel prescription, by adopting the de Sitter metric in combination with its Levi-Civita connection. For this momentum space, which had not been studied before, we derive several results which should be valuable for future studies. Still in Section $\mathrm{V}$ we derive the law of composition of momenta that would be attributed to "proper de Sitter momentum space" if adopting the prescription alternative to the one we are here advocating.

Some of the main results of our analysis are located in Section VI. There we establish and we characterize the (DSR-)relativistic properties of the novel proper de Sitter momentum space. And also for the already well known $\kappa$-momentum space we analyze relativistic properties in greater depth than ever before, establishing more firmly its DSR-relativistic compatibility but also highlighting more vividly the peculiarities of the associated relativistic theory.

While most of our results are obtained adopting a choice of coordinatization of momentum space, the findings about DSR-relativistic compatibility of our de Sitter momentum spaces are independent of such choices. This is shown in Section VII. We do not settle the issue of whether or not theories on curved momentum space are (or should be) diffeomorphism invariant, but we find that specifically the property of momentum spaces of being relativistically compatible (i.e. compatible with the formulation of DSR-relativistic theories on that momentum space) is a "geometric property", indeed diffeomorphism invariant. Section VIII summarizes our findings and offers some expectations for the possible development of this research area.

\section{GEOMETRY OF MOMENTUM SPACE FROM ON-SHELL RELATION AND MOMENTUM CONSERVATION}

The conceptual challenge which is at center stage in our study is the one of providing a suitable geometrical interpretation of relativistic kinematics. Of course, this is not a particularly interesting challenge in the presently adopted description of relativistic kinematics, for which it is evidently appropriate to adopt an interpretation based on a Minkowskian momentum space:

$$
\left\{\begin{array}{l}
m^{2}=p_{0}^{2}-|\vec{p}|^{2}=\eta^{\mu \nu} p_{\mu} p_{\nu} \\
p=q+k
\end{array}\right.
$$

where, for definiteness, we specialized the conservation law to the case of a three-particle event (a two-body decay).

But it does turn into a highly nontrivial challenge when contemplating, as done in part of the quantum-gravity literature, modifications of special-relativistic kinematics, with the on-shellness and the law of energy-momentum conservation taking in general the form

$$
\left\{\begin{array}{l}
m^{2}=d_{\ell}^{2}(p) \\
p=q \oplus_{\ell} k
\end{array}\right.
$$

where $d_{\ell}^{2}$ and $\oplus_{\ell}$ are functions of the components of the involved momenta and of the scale $\ell$, here assumed to be the inverse of the quantum-gravity scale (the dependence of $\oplus_{\ell}$ on $q$ and $k$ could be rendered more explicit in the notation by writing $p=q \oplus_{\ell}(q, k) k$ but we opt for leaving it implicit in order to keep our notation agile). 


\section{A. Geometrical Interpretation of the on-shell relation}

The conceptual perspective of the relative-locality framework [9, 10] provides an interpretation based on the geometry of momentum space for deformations of on-shellness and of momentum-conservation laws. The metric $g^{\mu \nu}$ on momentum space is linked to the on-shell relation while the affine connection on momentum space $\Gamma^{\lambda \mu}{ }_{\nu}$ is linked to the law of composition of momenta $\oplus_{\ell}$, which is the core ingredient of laws of conservation of energy-momentum.

According to Ref. [9] the link between on-shellness and metric on momentum space is to be established by describing $d_{\ell}^{2}(p)$ as distance of $p$ from the origin of momentum space, distance given in terms of the momentum-space metric. In formulas this means that

$$
m^{2}=d_{\ell}^{2}(p, 0)=\int d t \sqrt{g^{\mu \nu}\left(\gamma^{[A ; p]}(t)\right) \dot{\gamma}_{\mu}^{[A ; p]}(t) \dot{\gamma}_{\nu}^{[A ; p]}(t)}
$$

where $g^{\mu \nu}$ is the momentum-space metric and $\gamma^{[A ; p]}(t)$ is the metric geodesics connecting the point $p$ to the origin of momentum space. For the metric geodesics one has that

$$
\frac{d^{2} \gamma_{\lambda}^{[A]}(t)}{d t^{2}}+A_{\lambda}^{\mu \nu} \frac{d \gamma_{\mu}^{[A]}(t)}{d t} \frac{d \gamma_{\nu}^{[A]}(t)}{d t}=0
$$

where $A_{\lambda}^{\mu \nu}$ is the Levi-Civita connection.

Note that in general the relative-locality framework allows (also see the next subsection) for the adoption of an affine connection which may not be the Levi-Civita connection. Even in such cases the Levi-Civita connection $A^{\mu \nu}{ }_{\lambda}$ governs (4) (and therefore governs the on-shellness), while the affine connection $\Gamma_{\lambda}^{\mu \nu}$ governs the law of composition of momenta.

In addition to the metric geodesics (4), which evidently play a pivotal role in the relative-locality framework, we shall here also consider the possible role of connection geodesics

$$
\frac{d^{2} \gamma_{\lambda}(t)}{d t^{2}}+\Gamma_{\lambda}^{\mu \nu} \frac{d \gamma_{\mu}(t)}{d t} \frac{d \gamma_{\nu}(t)}{d t}=0
$$

\section{B. Standard geometrical interpretation of the Composition Law}

Ref. 9] also introduces an affine connection on momentum space, $\Gamma^{\mu \nu}{ }_{\lambda}$, through the following definition

$$
\Gamma_{\lambda}^{\mu \nu}(p)=-\left.\frac{\partial}{\partial q_{\mu}} \frac{\partial}{\partial k_{\nu}}\left(q \oplus_{\ell}^{[p]} k\right)_{\lambda}\right|_{q=k=p}
$$

where

$$
q \oplus_{\ell}^{[p]} k=p \oplus_{\ell}\left[\left(\ominus_{\ell} p \oplus_{\ell} q\right) \oplus_{\ell}\left(\ominus_{\ell} p \oplus_{\ell} k\right)\right]
$$

and $\ominus_{\ell}$ is the so-called "antipode" operation of $\oplus_{\ell}$, such that 1

$$
\left(\ominus_{\ell} p\right) \oplus_{\ell} p=0=p \oplus_{\ell}\left(\ominus_{\ell} p\right)
$$

As observed in Ref.[9], at least for the leading-order-in- $\ell$ approximation of (6), the composition rule $q \oplus_{\ell} k$ can be interpreted in terms of the parallel transport of $k$ along the geodesic connecting the origin of momentum space to $q$, i.e.

$$
\left(q \oplus_{\ell} k\right)_{\lambda} \simeq q_{\lambda}+k_{\alpha} \tau_{\lambda}^{\alpha}(q)
$$

where $\tau$ is the parallel transport operator, whose first-order expression is:

$$
\tau_{\lambda}^{\alpha}(q)=\delta_{\lambda}^{\alpha}-\Gamma_{\lambda}^{\beta \alpha}
$$

Note that this parallel transport is consistent with the following definition of the covariant derivative of a vector $V_{\alpha}$ :

$$
\nabla^{\lambda} V_{\alpha}=\partial^{\lambda} V_{\alpha}+\Gamma_{\alpha}^{\lambda \beta} V_{\beta}
$$

\footnotetext{
${ }^{1}$ Notice that in general the momentum-space affine connection may in some cases be such that $p \oplus_{\ell} k \neq k \oplus_{\ell} p$, but even in such cases one has that when $p \oplus_{\ell} k=0$ then also $k \oplus_{\ell} p=0$.
} 


\section{A novel geometrical interpretation of the Composition Law}

The geometrical interpretations of on-shellness and composition laws reviewed in the previous two subsections have already been adopted in several studies, and we shall refer to them as aspects of the "standard geometrical interpretation". For reasons that will become clearer as we go along we are going to consider here also an alternative geometrical interpretation. This novel geometrical interpretation still links the metric of momentum space and the on-shellness as described in Subsection [IA, but, building on a proposal first put forward preliminarily by Mercati [26], it adopts a description of the link between composition law and affine connection which is in general different from the one suggested by (6).

Our perspective on the geometrical interpretation of the composition law associates to the points $q$ and $k$ the connection geodesics $\gamma^{(q)}$ and $\gamma^{(k)}$ which connect them to the origin of momentum space. Then one introduces also a third curve $\bar{\gamma}(s)$, which we call the parallel transport of $\gamma^{(k)}(s)$ along $\gamma^{(q)}(t)$, such that for any given value $\bar{s}$ of the parameter $s$ one has that the tangent vector $\frac{d}{d s} \bar{\gamma}(\bar{s})$ is the parallel transport of the tangent vector $\frac{d}{d s} \gamma^{(k)}(\bar{s})$ along the geodesic connecting $\gamma^{(k)}(\bar{s})$ to $\bar{\gamma}(\bar{s})$. Then the composition law is defined as the extremal point of $\bar{\gamma}$, that is:

$$
q \oplus_{\ell} k=\bar{\gamma}(1)
$$

We also illustrate this prescription in Fig.1.

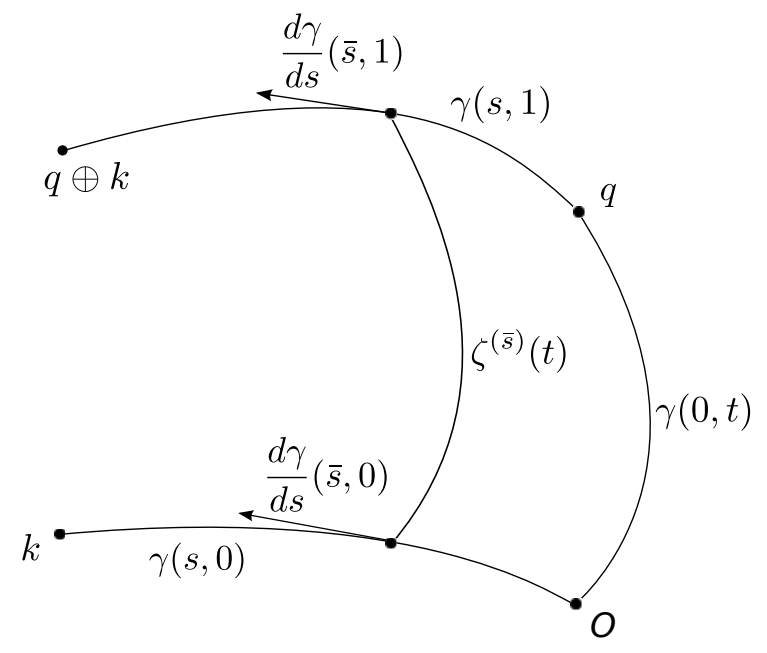

Figure 1. We determine the law of composition of momenta from the affine connection by associating to the points $q$ and $k$ of momentum space the connection geodesics $\gamma^{(q)}$ and $\gamma^{(k)}$ which connect them to the origin of momentum space. We then introduce a third curve $\bar{\gamma}(s)$, which we call the parallel transport of $\gamma^{(k)}(s)$ along $\gamma^{(q)}(t)$, such that for any given value $\bar{s}$ of the parameter $s$ one has that the tangent vector $\frac{d}{d s} \bar{\gamma}(\bar{s})$ is the parallel transport of the tangent vector $\frac{d}{d s} \gamma^{(k)}(\bar{s})$ along the geodesic connecting $\gamma^{(k)}(\bar{s})$ to $\bar{\gamma}(\bar{s})$. Then the composition law is defined as the extremal point of $\bar{\gamma}$, that is $q \oplus_{\ell} k=\bar{\gamma}(1)$.

A useful tool for setting up a computational approach based on this perspective is the introduction of a parametrized surface $\gamma(s, t)$ which is related to $\bar{\gamma}$ by $\gamma(s, 1)=\bar{\gamma}(s)$ and is constrained by the following boundary conditions:

$$
\left\{\begin{array}{l}
\gamma(s, 0)=\gamma^{(k)}(s) \\
\gamma(0, t)=\gamma^{(q)}(t)
\end{array}\right.
$$

The condition on the tangent vector of $\bar{\gamma}$ provides then a condition for $\gamma(s, t)$ : we want the vector $\frac{d \gamma}{d s}(s, t)$ to be the parallel transport of the vector $\frac{d \gamma}{d s}(s, 0)$ along the geodesic $\zeta^{(s)}$, defined for any $s$, connecting $\gamma(s, 0)$ to $\gamma(s, 1)$. This gives

$$
\frac{d}{d t} \frac{d}{d s} \gamma_{\lambda}(s, t)+\Gamma_{\lambda}^{\mu \nu}\left(\zeta_{s}(t)\right) \frac{d \zeta_{\mu}^{(s)}(t)}{d t} \frac{d \gamma_{\nu}(s, t)}{d s}=0
$$


where $\zeta^{(s)}(t)$ is the geodesic connecting $\gamma(s, 0)$ to $\gamma(s, 1)$, i.e.

$$
\left\{\begin{array}{l}
\frac{d^{2}}{d t^{2}} \zeta_{\lambda}^{(s)}(t)+\Gamma_{\lambda}^{\mu \nu}\left(\zeta^{(s)}(t)\right) \frac{d \zeta_{\mu}^{(s)}(t)}{d t} \frac{d \zeta_{\nu}^{(s)}(t)}{d t}=0 \\
\zeta^{(s)}(0)=\gamma^{(k)}(s) \\
\zeta^{(s)}(1)=\gamma(s, 1)
\end{array}\right.
$$

The composition law is then defined as:

$$
q \oplus_{\ell} k=\gamma(1,1)
$$

We note that equation 13 can also be written (in an equivalent but more explicitly covariant way) as

$$
\frac{d \zeta_{\mu}(s, t)}{d t} \nabla_{\Gamma}^{\mu} \frac{d \gamma_{\lambda}(s, t)}{d s}=0
$$

where $\nabla_{\Gamma}$ is the covariant derivative associated to the connection $\Gamma$. In this form it is made explicit the fact that the covariant derivative of the vector $\frac{d \gamma}{d s}(s, t)$ vanishes along the direction of the tangent vector $\frac{d \zeta}{d t}$.

We will show in appendix $\mathrm{D}$ that the connection $\Gamma^{\mu \nu}{ }_{\lambda}(p)$ implicitly defined in our framework by $(13)$ can be extracted from a "translated composition law" $\oplus_{\ell}^{[p]}$ in the following way

$$
\Gamma_{\lambda}^{\mu \nu}(p)=-\left.\frac{\partial}{\partial q_{\mu}} \frac{\partial}{\partial k_{\nu}}\left(q \oplus_{\ell}^{[p]} k\right)_{\lambda}\right|_{q=k=p}
$$

For this to hold one must define the translated composition law $\oplus_{\ell}^{[p]}$ through the same kind of construction that we have described above, but with each momentum associated to the geodesic connecting it to the "subtraction point" $p$ instead of the origin. In formulas this is the prescription that

$$
\left\{\begin{array}{l}
\frac{d}{d t} \frac{d}{d s} \gamma_{\lambda}^{[p]}(s, t)+\Gamma^{\mu \nu}{ }_{\lambda}\left(\zeta_{s}(t)\right) \frac{d \zeta_{\mu}^{(s)}(t)}{d t} \frac{d \gamma_{\nu}^{[p]}(s, t)}{d s}=0 \\
\gamma^{[p]}(s, 0)=\gamma^{(k, p)}(s) \\
\gamma^{[p]}(0, t)=\gamma^{(q, p)}(t) \\
\gamma^{[p]}(1,1)=q \oplus_{\ell}^{[p]} k \\
\frac{d^{2}}{d t^{2}} \zeta_{\lambda}^{(s)}(t)+\Gamma^{\mu \nu}{ }_{\lambda}\left(\zeta^{(s)}(t)\right) \frac{d \zeta_{\mu}^{(s)}(t)}{d t} \frac{d \zeta_{\nu}^{(s)}(t)}{d t}=0 \\
\zeta^{(s)}(0)=\gamma^{(q, p)}(s) \\
\zeta^{(s)}(1)=\gamma^{[p]}(s, 1)
\end{array}\right.
$$

where $\gamma^{(q, p)}$ is the geodesic defined by:

$$
\left\{\begin{array}{l}
\frac{d^{2}}{d t^{2}} \gamma_{\lambda}^{(q, p)}(t)+\Gamma^{\mu \nu}{ }_{\lambda}\left(\gamma^{(q, p)}(t)\right) \frac{d \gamma_{\mu}^{(q, p)}(t)}{d t} \frac{d \gamma_{\nu}^{(q, p)}(t)}{d t}=0 \\
\gamma^{(q, p)}(0)=p \\
\gamma^{(q, p)}(1)=q
\end{array}\right.
$$

and a similar formula holds also for $\gamma^{(k, p)}$.

\section{Aside on an ambiguity in the link from composition law to affine connection}

Since one of the issues that we are keeping in focus concerns the nature of the link between affine connection and law of composition of momenta it is important for us to stress an aspect of ambiguity of this link which arises when the composition law is noncommutative, $p \oplus_{\ell} k \neq k \oplus_{\ell} p$. This ambiguity is present both with the standard geometricinterpretation prescription, here coded in Eq.(6), and with the novel geometric interpretation we introduced through Eqs. 132- 15 . The fact here relevant is that the physics of the composition law of course resides in the composition law itself, not in its derivatives. As a result of this there is no priority of the definition (6), which is

$$
\Gamma_{\lambda}^{\mu \nu}(p)=-\left.\frac{\partial}{\partial q_{\mu}} \frac{\partial}{\partial k_{\nu}}\left(q \oplus_{\ell}^{[p]} k\right)_{\lambda}\right|_{q=k=p}
$$


over the possible alternative

$$
\Gamma_{\lambda}^{\mu \nu}(p)=-\left.\frac{\partial}{\partial k_{\mu}} \frac{\partial}{\partial q_{\nu}}\left(q \oplus_{\ell}^{[p]} k\right)_{\lambda}\right|_{q=k=p} .
$$

The same issue can be described by noticing that given the definition 20 one still has the freedom of specifying which one of the upper indices of $\Gamma^{\mu \nu}{ }_{\lambda}$ is the differential index. For differential index we mean the index of the connection that in the expression of the covariant derivative is paired with the index of the partial derivative. The ambiguity then is associated with the possibility of structuring the covariant derivative either as

$$
\nabla_{(1)}^{\lambda} V^{\mu}=\partial^{\lambda} V^{\mu}-\Gamma_{\nu}^{\lambda \mu} V^{\nu}
$$

or as

$$
\nabla_{(2)}^{\lambda} V^{\mu}=\partial^{\lambda} V^{\mu}-\Gamma_{\nu}^{\mu \lambda} V^{\nu}
$$

where $V^{\mu}$ is an arbitrary vector.

Evidently the same ambiguity also affects the novel prescription which we are here introducing. This can be noticed by looking at Eq. (14): just like one can describe $q \oplus_{\ell} k$ in terms of the parallel transport of $\gamma^{(k)}$ along $\gamma^{(q)}$ it would of course be also possible to describe $q \oplus_{\ell} k$ in terms of the parallel transport of $\gamma^{(q)}$ along $\gamma^{(k)}$. This means that one can obtain the composition law associated to a given affine connection by following the same geometrical construction we illustrated in previous section, but modifying accordingly the boundary conditions in such a way that the role of $\gamma^{(k)}$ and $\gamma^{(q)}$ is exchanged. The end result is

$$
\left\{\begin{array}{l}
\frac{d}{d t} \frac{d}{d s} \gamma_{\lambda}(s, t)+\Gamma_{\lambda}^{\mu \nu}\left(\zeta_{s}(t)\right) \frac{d \zeta_{\mu}^{(s)}(t)}{d t} \frac{d \gamma_{\nu}(s, t)}{d s}=0 \\
\gamma(s, 0)=\gamma^{(q)}(s) \\
\gamma(0, t)=\gamma^{(k)}(t) \\
\gamma(1,1)=q \oplus_{\ell} k \\
\frac{d^{2}}{d t^{2}} \zeta_{\lambda}^{(s)}(t)+\Gamma_{\lambda \nu}^{\mu \nu}\left(\zeta^{(s)}(t)\right) \frac{d \zeta_{\mu}^{(s)}(t)}{d t} \frac{d \zeta_{\nu}^{(s)}(t)}{d t}=0 \\
\zeta^{(s)}(0)=\gamma^{(q)}(s) \\
\zeta^{(s)}(1)=\gamma(s, 1)
\end{array}\right.
$$

Clearly this ambiguity only affects cases where the connection is torsionful. Most importantly, it only concerns the geometric interpretation of the composition law, rather than the composition law itself, and therefore it has no implications for physics. It is however something that needs to be dealt with if one is interested in finding general implications of the geometry of momentum space for physics (since indeed the ambiguity is such that somewhat different geometries of momentum space could be associated to the same physical theory). An example of how this might come to be relevant is provided by the case of studies intending to establish some general implications of nonmetricity of momentum space for the physics of the relevant theories: keeping fixed the on-shellness and the composition law (which specify the physical content of the theory) one can easily find cases such that the ambiguity here highlighted involves a possible description in terms of a momentum space without nonmetricity and a possible description in terms of a momentum space with nonmetricity.

\section{DIFFERENCES BETWEEN THE STANDARD AND OUR NOVEL PRESCRIPTION FOR THE GEOMETRY OF MOMENTUM SPACE}

Since a large part of what we are here reporting concerns a novel proposal for the geometric interpretation of momentum-space kinematics, we find appropriate to pause in this section for some comments on two related issues:

- the strength that in general one can expect for the link between the momentum-space affine connection and the form of the composition law;

- the differences between the standard geometric-interpretation prescription, here coded in Eq.60), and the novel geometric interpretation we introduced through Eqs. 13 - -15 .

We confine our exploration of these issues up to second order in the deformation scale, which already exposes several interesting structures and allows us to keep the analysis completely explicit. And we assume the composition law has the natural property $k \oplus_{\ell} 0=k$. Our starting point then is the following form of the most general composition law at second order in the deformation scale $\ell$ :

$$
\left(q \oplus_{\ell} k\right)_{\lambda}=q_{\lambda}+k_{\lambda}+\ell X_{\lambda}^{\alpha \beta} q_{\alpha} k_{\beta}+\frac{\ell^{2}}{2} Y_{\lambda}^{\alpha \beta \gamma} q_{\alpha} q_{\beta} k_{\gamma}+\frac{\ell^{2}}{2} Z_{\lambda}^{\alpha \beta \gamma} q_{\alpha} k_{\beta} k_{\gamma}
$$


The matrices $X, Y$ and $Z$ are to be determined experimentally. Evidently we are free to choose any desired geometrical interpretation of composition laws, since this geometric interpretation is not in itself observable (the physics of the composition law is indeed contained in these matrices $X, Y$ and $Z$ ). But still the choice of geometrical interpretation could have tangible consequences, since the correct choice of geometrical interpretation might prove to be particularly advantageous for characterizing the observable properties of the composition law. A geometrical interpretation of kinematics on momentum space cannot be right or wrong, but could nonetheless be judged on the basis of its usefulness (or lack thereof). An important question then is to what extent one can associate an affine connection on momentum space to one of these composition laws. Is there always a suitable affine connection for any composition law? And, if so, is there a unique affine connection that "fits", according to a given prescription of geometrical interpretation?

In exploring these questions one should possibly look for simple links between properties of the affine connection and properties of the composition law. Candidate simple links of this sort could be for example linking torsion to noncommutativity of the composition law and/or linking curvature to non-associativity of the composition law. Furthermore in Refs.[18, 27] the possibility has been raised that perhaps the property of kinematics on momentum space of being (DSR-)relativistic, which is not in general assured [18, might itself admit a geometric description.

Keeping these issues in the background let us start the analysis from contributions to momentum composition at first order in the deformation scale. It is easy to check that, in the notation of 23 , both the previously standard prescription and the novel prescription we are here introducing agree on specifying the corresponding leading-order contribution to the affine connection as follows

$$
\Gamma_{\lambda}^{\mu \nu}(0)=\ell X_{\lambda}^{\mu \nu}
$$

It is very hard to imagine any natural alternative choice for this leading-order relationship. And notice that the matrix $X$ and the form of the affine connection in the origin of momentum space, $\Gamma(0)$, have the same number of components.

The naturalness of the leading-order prescription 24 is very clearly not matched by the situation encountered at second order. In order to see this let us expand the connection as

$$
\Gamma_{\lambda}^{\mu \nu}(p)=\Gamma_{\lambda}^{\mu \nu}(0)+\left.p_{\theta} \partial^{\theta} \Gamma_{\lambda}^{\mu \nu}(p)\right|_{p=0}
$$

which also implies that $\left.\partial^{\theta} \Gamma^{\mu \nu}{ }_{\lambda}(p)\right|_{p=0}$ is of second order in the scale $\ell$.

While there appears to be a unique natural link that can be established between the matrix $X$ characteristic of the leading-order deformation of the composition law and $\Gamma(0)$, one can imagine several ways for introducing a link between the matrices $Y, Z$ characteristic of the next-to-leading-order deformation of the composition law and $\left.\partial \Gamma\right|_{p=0}$. The first immediate difficulty in this respect is given by the fact that $Y$ and $Z$ have more degrees of freedom than $\partial \Gamma$. In fact, considering for simplicity the $1+1$ dimensional case, we note that $Y$ and $Z$ have a total number of 24 independent components (they are, respectively, symmetric in the first two and in the last two indices), while $\partial \Gamma$ can handle a maximum of 16 components. This means that the space of possible connections is smaller than the space of possible composition laws. So, one shall inevitably have one of two options:

either only a subset of the possible composition laws will admit an associated affine connection

or all possible composition laws will admit an associated affine connection but different composition laws will be mapped into the same connection.

This second option is the one that applies to the "standard geometric interpretation", here coded in Eq. (6). We can quickly show this, working at second order in $\ell$. We start of course from (6) which we note down again here for convenience:

$$
\Gamma_{\lambda}^{\mu \nu}(p)=-\left.\frac{\partial}{\partial q_{\mu}} \frac{\partial}{\partial k_{\nu}}\left(q \oplus_{\ell}^{[p]} k\right)_{\lambda}\right|_{q=k=p}
$$

Substituting here the general form of the composition law (23), using the expression 77 and with the condition 24 , one easily establishes, up to second order in $\ell$, that

$$
\Gamma_{\lambda}^{\mu \nu}(p) \simeq \Gamma_{\lambda}^{\mu \nu}(0)+\Gamma_{\lambda}^{\mu \beta}(0) \Gamma_{\beta}^{\gamma \nu}(0) p_{\gamma}+\Gamma_{\lambda}^{\alpha \nu}(0) \Gamma_{\alpha}^{\gamma \mu}(0) p_{\gamma}-\Gamma_{\lambda}^{\alpha \beta}(0) \Gamma_{\beta}^{\mu \nu}(0) p_{\alpha}-\ell^{2} Z_{\lambda}^{\alpha \mu \nu} p_{\alpha}
$$

Then expanding the connection as in 25 one gets

$$
\left.\partial^{\theta} \Gamma_{\lambda}^{\mu \nu}(p)\right|_{p=0}=\Gamma_{\lambda}^{\mu \beta}(0) \Gamma_{\beta}^{\gamma \nu}(0)+\Gamma_{\lambda}^{\alpha \nu}(0) \Gamma_{\alpha}^{\gamma \mu}(0)-\Gamma_{\lambda}^{\gamma \beta}(0) \Gamma_{\beta}^{\mu \nu}(0)-\ell^{2} Z_{\lambda}^{\gamma \mu \nu}
$$

So we see that the possible role played in the composition law by the matrix $Y$ is completely neglected by the standard prescription (26). Indeed, using the above equation, we can rewrite 23 so that the dependence of the most 
general composition law 23) on the connection, according to the standard prescription (26), is explicit:

$$
q \oplus_{\ell} k=q_{\lambda}+k_{\lambda}-\ell \bar{\Gamma}^{\alpha \beta}{ }_{\lambda} q_{\alpha} k_{\beta}+\frac{\ell^{2}}{2} Y_{\lambda}^{\alpha \beta \gamma} q_{\alpha} q_{\beta} k_{\gamma}+\frac{\ell^{2}}{2}\left(\partial^{\theta} \bar{\Gamma}_{\lambda}^{\mu \nu}-\bar{\Gamma}_{\lambda}^{\beta \theta} \bar{\Gamma}_{\theta}^{\alpha \gamma}-\bar{\Gamma}_{\lambda}^{\theta \gamma} \bar{\Gamma}_{\theta}^{\alpha \beta}+\bar{\Gamma}_{\lambda}^{\alpha \theta}{ }_{\lambda} \bar{\Gamma}^{\beta \gamma}{ }_{\theta}\right) q_{\alpha} k_{\beta} k_{\gamma}
$$

where we found convenient to render explicit the powers of $\ell$ through the definitions

$$
\left\{\begin{array}{l}
\ell \bar{\Gamma}^{\mu \nu}{ }_{\lambda} \equiv \Gamma^{\mu \nu}{ }_{\lambda}(0) \\
\left.\ell^{2} \partial^{\theta} \bar{\Gamma}^{\mu \nu}{ }_{\lambda} \equiv \partial^{\theta} \Gamma^{\mu \nu}{ }_{\lambda}(p)\right|_{p=0}
\end{array}\right.
$$

This shows that according to the standard prescription (26), the composition law is not completely specified by a given connection, as we have the freedom of arbitrarily choosing the 12 components of $Y$. So the way in which the difference in the number of components of $(Y, Z)$ and $\Gamma$ is handled within the standard prescription (26) is to discard completely the contribution given by $Y$. And notice that this does not cover even all of the degrees of freedom of $\partial \Gamma$, which are 16: those are used to specify only the matrix $Z$ with its 12 degrees of freedom. The degrees of freedom of the connection which, according to (26), are not relevant for the description of any composition law are easily identified, using (28) and exploiting the symmetries of $Z$. Indeed this leads to the requirement

$$
\left.\partial^{\theta} \Gamma_{\lambda}^{[\mu \nu]}(p)\right|_{p=0}=\Gamma_{\lambda}^{[\mu \beta}(0) \Gamma_{\beta}^{\gamma \nu]}(0)+\Gamma_{\lambda}^{\alpha[\nu}(0) \Gamma_{\alpha}^{\gamma \mu]}(0)-\Gamma_{\lambda}^{\gamma \beta}(0) \Gamma_{\beta}^{[\mu \nu]}(0)
$$

which in $1+1$ dimensions is a set of 4 independent equations on $\partial \Gamma$. So we find that the standard interpretation of the composition law encoded in (26) at second order in the scale $\ell$ provides a map between the set of possible $Z$ and the set of connections which satisfy the requirement (31). This is a noteworthy difference between the standard interpretation (26) and the novel interpretation here introduced in (13)- 15), which does not involve any limitation of the form of the connections.

Since part of our focus here is also on the possibility of commutative (and yet deformed) composition laws, it is important to notice that this peculiar features of the standard prescription (26) are also responsible for the fact that symmetric connections can correspond to noncommutative composition laws.

$$
\Gamma_{\lambda}^{\mu \nu}(p)=\Gamma_{\lambda}^{\nu \mu}(p) \nRightarrow q \oplus_{\ell} k=k \oplus_{\ell} q
$$

Let us now show that the novel prescription of geometrical interpretation of the composition law which we are here proposing, introduced through Eqs. (13)-(15), has completely complementary properties with respect to the standard prescription. Indeed our novel prescription applies only to a subset of composition laws, but for that subset establishes a one-to-one correspondence between form of the composition law and form of the affine connection. This is easily seen by making explicit at second order in $\ell$ the map established by our Eqs. 13)- 15 obtaining, as we show in the appendix C

$$
\left(q \oplus_{\ell} k\right)_{\lambda}=q_{\lambda}+k_{\lambda}-\ell \bar{\Gamma}^{\alpha \beta}{ }_{\lambda} q_{\alpha} k_{\beta}-\frac{\ell^{2}}{2} \partial^{\rho} \bar{\Gamma}^{\alpha \beta}{ }_{\lambda} q_{\alpha} k_{\beta}\left(q_{\rho}+k_{\rho}\right)+\frac{\ell^{2}}{2} \bar{\Gamma}_{\lambda}^{\alpha \beta}{ }_{\lambda} \bar{\Gamma}_{\alpha \delta}{ }_{\alpha} q_{\gamma} k_{\delta} k_{\beta}+\frac{\ell^{2}}{2} \bar{\Gamma}^{\alpha \beta}{ }_{\lambda} \bar{\Gamma}^{\gamma \delta}{ }_{\beta} q_{\alpha} q_{\gamma} k_{\delta}
$$

Since commutative composition laws are one of the main motivations for our analysis, let us start by noticing that, unlike the standard prescription (26), our new geometric-interpretation prescription, here summarized in (33), is such that symmetric connections are mapped into commutative composition laws:

$$
\begin{gathered}
\Gamma^{\mu \nu}{ }_{\lambda}(p)=\Gamma^{\nu \mu}{ }_{\lambda}(p) \\
\Downarrow{ }_{\lambda} \\
q \oplus_{\ell} k=q_{\lambda}+k_{\lambda}-\ell \bar{\Gamma}^{\alpha \beta}{ }_{\lambda} q_{\alpha} k_{\beta}-\frac{\ell^{2}}{2} \partial^{\rho} \bar{\Gamma}^{\alpha \beta}{ }_{\lambda} q_{\alpha} k_{\beta}\left(q_{\rho}+k_{\rho}\right)+\frac{\ell^{2}}{2} \bar{\Gamma}^{\alpha \beta}{ }_{\lambda} \bar{\Gamma}^{\gamma \delta}{ }_{\alpha} q_{\gamma} k_{\delta} k_{\beta}+\frac{\ell^{2}}{2} \bar{\Gamma}^{\alpha \beta}{ }_{\lambda} \bar{\Gamma}^{\gamma \delta}{ }_{\beta} q_{\alpha} q_{\gamma} k_{\delta}= \\
=q_{\lambda}+k_{\lambda}-\ell \bar{\Gamma}^{\beta \alpha}{ }_{\lambda} q_{\alpha} k_{\beta}-\frac{\ell^{2}}{2} \partial^{\rho} \bar{\Gamma}^{\beta \alpha}{ }_{\lambda} q_{\alpha} k_{\beta}\left(q_{\rho}+k_{\rho}\right)+\frac{\ell^{2}}{2} \bar{\Gamma}^{\beta \alpha}{ }_{\lambda} \overline{\bar{\Gamma}}^{\delta \gamma}{ }_{\alpha} q_{\gamma} k_{\delta} k_{\beta}+\frac{\ell^{2}}{2} \bar{\Gamma}^{\beta \alpha}{ }_{\lambda} \overline{\bar{\Gamma}}^{\delta \gamma}{ }_{\beta} q_{\alpha} q_{\gamma} k_{\delta}=k \oplus_{\ell} q
\end{gathered}
$$

From a wider perspective one should notice that, as shown in (33), according to our prescription the affine connection on momentum space reflects both properties of the matrix $Y$ and properties of the matrix $Z$ (the two matrices characterizing in our notation the composition law at second order in $\ell$ ), whereas as shown above the standard prescription (26) is such that only the matrix $Z$ leaves a trace in the form of the affine connection. However, as also noticed above, the combined number of degrees of freedom of the matrices $Y$ and $Z$ is larger than the number of degrees of freedom of the affine connection at second order in $\ell$. So our prescription establishes a dependence of the affine connection on both the matrix $Y$ and the matrix $Z$ but cannot code in the affine connection the most general form of the composition law. In order to explore this issue we compare the second-order expansion (33) with the one 
holding for the most general composition law, Eq. 23], and we obtain from the parts quadratic in $q$ and from the parts quadratic in $k$ the following system of equations

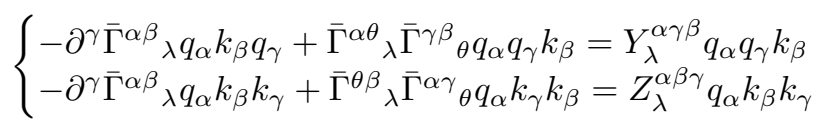

These two equations, taken separately, require

$$
\left\{\begin{array}{l}
\partial^{\gamma} \bar{\Gamma}_{\lambda}^{\alpha \beta}=-Y_{\lambda}^{\alpha \gamma \beta}+\bar{\Gamma}_{\lambda}^{(\alpha \theta}{ }_{\lambda} \bar{\Gamma}^{\gamma) \beta}{ }_{\theta}+t_{\lambda}^{\alpha \beta \gamma} \\
\partial^{\gamma} \bar{\Gamma}_{\lambda}^{\alpha \beta}=-Z_{\lambda}^{\alpha \beta \gamma}+\bar{\Gamma}_{\lambda}^{\theta(\beta}{ }_{\lambda} \bar{\Gamma}^{\alpha \gamma)}{ }_{\theta}+s_{\lambda}^{\alpha \beta \gamma}
\end{array}\right.
$$

where $t$ is antisymmetric in $\alpha$ and $\gamma$ while $s$ is antisymmetric in $\beta$ and $\gamma$. The fact that not all possible choices of the matrices $Y$ and $Z$ can be mapped into one of our affine connections is manifest in the fact that these two solutions are not in general compatible with each other. The solutions will be compatible with each other if and only if one requires that

$$
-Y_{\lambda}^{\alpha \gamma \beta}+\bar{\Gamma}_{\lambda}^{(\alpha \theta} \bar{\Gamma}_{\theta}^{\gamma) \beta}+t^{\alpha \beta \gamma}=-Z_{\lambda}^{\alpha \beta \gamma}+\bar{\Gamma}_{\lambda}^{\theta(\beta}{ }_{\lambda}^{\alpha \gamma)}{ }_{\theta}+s^{\alpha \beta \gamma}
$$

We observe that by taking the sum of cyclic permutations of the upper free indices on both sides this may be turned into the requirement that

$$
\operatorname{Cycl}\left(-Y_{\lambda}^{\alpha \gamma \beta}+\bar{\Gamma}_{\lambda}^{(\alpha \theta} \bar{\Gamma}_{\theta}^{\gamma) \beta}\right)=\operatorname{Cycl}\left(-Z_{\lambda}^{\alpha \beta \gamma}+\bar{\Gamma}_{\lambda}^{\theta(\beta} \bar{\Gamma}_{\theta}^{\alpha \gamma)}\right)
$$

where we introduced $C y c l$ defined as

$$
\operatorname{Cycl}\left(W_{\lambda}^{\alpha \beta \gamma}\right) \equiv W_{\lambda}^{\alpha \beta \gamma}+W_{\lambda}^{\gamma \alpha \beta}+W_{\lambda}^{\beta \gamma \alpha}
$$

Furthermore, it takes some simple algebra to verify that, if the cyclic condition (37) holds, then a solution of (36) exists and is given by:

$$
\left\{\begin{array}{l}
t^{\alpha \beta \gamma}=\frac{2}{3} \tilde{Y}^{[\alpha \beta \gamma]}-\frac{4}{3} \tilde{Z}^{[\alpha \beta \gamma]} \\
s^{\alpha \beta \gamma}=\frac{2}{3} \tilde{Z}^{[\gamma \alpha \beta]}-\frac{4}{3} \tilde{Y}^{[\gamma \alpha \beta]}
\end{array}\right.
$$

where we defined

$$
\left\{\begin{array}{l}
\tilde{Y}_{\lambda}^{\alpha \beta \gamma}=Y_{\lambda}^{\alpha \beta \gamma}-\Gamma^{(\alpha \theta}{ }_{\lambda} \Gamma^{\beta) \gamma} \theta \\
\tilde{Z}_{\lambda}^{\alpha \beta \gamma}=Z_{\lambda}^{\alpha \beta \gamma}-\Gamma^{\theta\left({ }_{\lambda} \Gamma^{\alpha \beta)} \theta\right.}
\end{array}\right.
$$

Inserting the expressions $(39)$ for $s$ and $t$ into the system of equations (35) and recalling (25) we find that the connection as a function of $Y$ and $Z$ can be given in the forms :

$$
\left\{\begin{array}{l}
\Gamma_{\lambda}^{\alpha \beta}(p)=\ell \bar{\Gamma}_{\lambda}^{\alpha \beta}+\ell^{2}\left(-\tilde{Y}_{\lambda}^{\alpha \gamma \beta}+\frac{2}{3} \tilde{Y}^{[\alpha \beta \gamma]}-\frac{4}{3} \tilde{Z}^{[\alpha \beta \gamma]}\right) p_{\gamma} \\
\Gamma_{\lambda}^{\alpha \beta}(p)=\ell \bar{\Gamma}_{\lambda}^{\alpha \beta}+\ell^{2}\left(-\tilde{Z}_{\lambda}^{\alpha \beta \gamma}+\frac{2}{3} \tilde{Z}^{[\gamma \alpha \beta]}-\frac{4}{3} \tilde{Y}^{[\gamma \alpha \beta]}\right) p_{\gamma}
\end{array}\right.
$$

which are evidently equivalent to each other because of (37). These (41) are the analogue, for our construction of the composition law, of equation (6); they allow us to associate at second order in $\ell$ a connection $\Gamma$ to any given composition law satisfying the cyclic condition (37).

We can characterize the cyclic condition 37 more vividly by rewriting it as the following property that, at quadratic order 2 in $\ell$, needs to be imposed on the composition law:

$$
\operatorname{Cycl}_{\oplus_{\ell}}\left\{\left(\ominus_{\ell} q \oplus_{\ell}\left(k \oplus_{\ell} q\right)\right)_{\lambda}\right\}=\operatorname{Cycl}_{\oplus_{\ell}}\left\{\left(\left(\ominus_{\ell} q \oplus_{\ell} k\right) \oplus_{\ell} q\right)_{\lambda}\right\}
$$

\footnotetext{
2 Eq. 42 is easily verified by expanding it at second order in $\ell$. Also note that since Eq. 37. characterizes only a second-order-in- $\ell$ condition, also Eq. 42 must be viewed as here determined only up to quadratic order in $\ell$ (in particular one could replace $\ominus_{\ell} q$ with $\oplus_{\ell} q$ in 42 and still get a relation equivalent to 37 at quadratic order in $\ell$ ).
} 
where Cycl $_{\oplus_{\ell}}$ is defined by

$$
\left\{\begin{array}{l}
\operatorname{Cycl}_{\oplus_{\ell}}\left\{\left(p \oplus_{\ell}\left(k \oplus_{\ell} q\right)\right)_{\lambda}\right\}=\left(p \oplus_{\ell}\left(k \oplus_{\ell} q\right)\right)_{\lambda}+\left(q \oplus_{\ell}\left(p \oplus_{\ell} k\right)\right)_{\lambda}+\left(k \oplus_{\ell}\left(q \oplus_{\ell} p\right)\right)_{\lambda} \\
\operatorname{Cycl}_{\oplus_{\ell}}\left\{\left(\left(p \oplus_{\ell} k\right) \oplus_{\ell} q\right)_{\lambda}\right\}=\left(\left(p \oplus_{\ell} k\right) \oplus_{\ell} q\right)_{\lambda}+\left(\left(q \oplus_{\ell} p\right) \oplus_{\ell} k\right)_{\lambda}+\left(\left(k \oplus_{\ell} q\right) \oplus_{\ell} p\right)_{\lambda}
\end{array}\right.
$$

We also observe that, focusing again on the $1+1 \mathrm{D}$ case, one has that the condition for sums of cyclic permutations (37) corresponds to a set of 8 independent conditions. The matrices $Y$ and $Z$ start off with a total of 24 degrees of freedom, and after this 8 conditions we are left with 16 degrees of freedom which exactly matches the number of degrees of freedom of the quadratic-in- $\ell$ part of the affine connection. This should be contrasted to the fact observed above that only 12 degrees of freedom of the composition law (the ones of the matrix $Z$ ) leave a trace in the affine connection according to the standard prescription (26).

\section{TWO $\kappa$-DE SITTER MOMENTUM SPACES}

The possibility which has been so far most studied [19, 28 30, within the relative-locality framework is the one of the $\kappa$-dS (" $\kappa$-de Sitter") momentum space. This is based on a form of on-shellness and a form of the law of composition of momenta inspired by the $k$-Poincaré Hopf algebra [31, 32, which had already been of interest from the quantum-gravity perspective for independent reasons [13, 33, 35].

As most previous works on this possibility [28, 29] we shall focus on the $1+1$-dimensional $\kappa$-dS momentum space, which allows us to discuss the key conceptual features in a slightly simplified context (with respect to the $3+1$ dimensional case). For the $1+1$-dimensional $\kappa$-dS momentum space the metric is a de Sitter metric,

$$
g^{\mu \nu}(p)=\left(\begin{array}{cc}
1 & 0 \\
0 & -e^{2 \ell p_{0}}
\end{array}\right)
$$

while the composition law has the form [28, 29]

$$
\left\{\begin{array}{l}
\left(q \oplus_{\ell} k\right)_{0}=q_{0}+k_{0} \\
\left(q \oplus_{\ell} k\right)_{1}=q_{1}+k_{1} e^{-\ell p_{0}}
\end{array}\right.
$$

We note down the antipode of this composition law

$$
\left\{\begin{array}{l}
\left(p \oplus_{\ell}\left(\ominus_{\ell} p\right)\right)_{0}=p_{0}+\ominus_{\ell} p_{0}=0 \rightarrow \ominus_{\ell} p_{0}=-p_{0} \\
\left(p \oplus_{\ell}\left(\ominus_{\ell} p\right)\right)_{1}=p_{1}+\ominus_{\ell} p_{1} e^{-\ell p_{0}}=0 \rightarrow \ominus_{\ell} p_{1}=-p_{1} e^{\ell p_{0}}
\end{array}\right.
$$

which in fact is such that $\ominus_{\ell} p \oplus_{\ell} p=0=p \oplus_{\ell}\left(\ominus_{\ell} p\right)$.

And we notice that the $\kappa$-dS composition law is associative

$$
\left(p \oplus_{\ell} q\right) \oplus_{\ell} k=p \oplus_{\ell}\left(q \oplus_{\ell} k\right) .
$$

For reasons that will be clearer in the following let us also observe that evidently for associative composition laws one has that the so-called "left loop inverse rule" and "right loop inverse rule" [36] apply:

$$
\begin{aligned}
& q \oplus_{\ell}\left(\ominus_{\ell} q \oplus_{\ell} p\right)=p \\
& \left(p \oplus_{\ell} \ominus_{\ell} q\right) \oplus_{\ell} q=p .
\end{aligned}
$$

Using the prescription summarized in the previous section (Eq. (3)) one easily finds that the de Sitter metric on momentum space leads to the following on-shell relation:

$$
m^{2}=d_{\ell}(p, 0)=\frac{1}{\ell} \operatorname{Arccosh}\left[\operatorname{Cosh}\left[\ell p_{0}\right]-\frac{\ell^{2}}{2} p_{1}^{2} e^{\ell p_{0}}\right]
$$

We notice that this too matches results in the $\kappa$-Poincaré literature, since it is evidently related to the " $\kappa$-deformed mass Casimir" customarily written as

$$
\operatorname{Cosh}[\ell m]=\operatorname{Cosh}\left[\ell d_{\ell}(p)\right]=\operatorname{Cosh}\left[\ell p_{0}\right]-\frac{\ell^{2}}{2} p_{1}^{2} e^{\ell p_{0}}
$$


This $\kappa$-dS momentum space is here of interest from several viewpoints. It is the most studied curved momentum space, and yet we have several points to contribute to its understanding (particularly concerning its relativistic properties, see later). Its composition law is noncommutative (as for all other momentum spaces so far studied in some detail), and so it serves as a reference of contrast to our proposal of a de Sitter momentum space with commutative composition law. And importantly this is an example of momentum space whose description is within reach of both the standard geometric-interpretation prescription, here coded in Eq. (6), and the novel geometric interpretation we introduced through Eqs. 13)-15). Since in this manuscript we often stress the differences between the standard geometric-interpretation prescription and our novel geometric interpretation it is important for us to also provide an example where no such differences are present. If all we were interested in were only cases like the $\kappa$-dS momentum space then there would be no difference between the standard geometric interpretation and our novel geometric interpretation.

For what concerns the description of the " $\kappa$-connection", i.e. the affine connection for the $\kappa$-dS momentum space, as obtained adopting the standard geometric-interpretation prescription, here coded in Eq.(6), we can rely on the previous analyses of Refs. [28, 29] establishing that

$$
\Gamma_{\nu}^{\lambda \mu}=\ell \delta_{0}^{\lambda} \delta_{1}^{\mu} \delta_{\nu}^{1}
$$

This result can be quickly reproduced starting from Eq.(6), which is

$$
\Gamma_{\lambda}^{\mu \nu}(p)=-\left.\frac{\partial}{\partial q_{\mu}} \frac{\partial}{\partial k_{\nu}}\left(q \oplus_{\ell}^{[p]} k\right)_{\lambda}\right|_{q=k=p}
$$

where in the $\kappa$-dS case we have that

$$
\left\{\begin{array}{l}
\left(q \oplus_{\ell}^{[p]} k\right)_{0}=\left(p \oplus_{\ell}\left[\left(\ominus_{\ell} p \oplus_{\ell} q\right) \oplus_{\ell}\left(\ominus_{\ell} p \oplus_{\ell} k\right)\right]\right)_{0}=q_{0}+k_{0}-p_{0} \\
\left(q \oplus_{\ell}^{[p]} k\right)_{1}=\left(p \oplus_{\ell}\left[\left(\ominus_{\ell} p \oplus_{\ell} q\right) \oplus_{\ell}\left(\ominus_{\ell} p \oplus_{\ell} k\right)\right]\right)_{1}=p_{1}+\left(q_{1}+k_{1}-2 p_{1}\right) e^{-\ell\left(q_{0}-p_{0}\right)}
\end{array}\right.
$$

From this it follows that

$$
\Gamma_{\lambda}^{\mu \nu}(p)=-\left.\frac{\partial}{\partial q_{\mu}} \frac{\partial}{\partial k_{\nu}}\left(q \oplus_{\ell}^{[p]} k\right)_{\lambda}\right|_{q=k=p}=\ell \delta_{0}^{\mu} \delta_{1}^{\nu} \delta_{\lambda}^{1}
$$

It is interesting to notice that this $1+1$-dimensional $\kappa$-dS momentum space has de Sitter metric (constant curvature $\left.-2 \ell^{2}\right)$ and affine connection which instead has no curvature:

$$
F_{\lambda}^{\mu \beta \alpha}=\partial^{\mu} \Gamma_{\lambda}^{\beta \alpha}-\partial^{\beta} \Gamma_{\lambda}^{\mu \alpha}+\Gamma_{\lambda}^{\mu \tau} \Gamma_{\tau}^{\beta \alpha}-\Gamma_{\lambda}^{\beta \tau} \Gamma_{\tau}^{\mu \alpha}=0
$$

\section{A. $\kappa$-dS composition law from $\kappa$-connection}

Our first task is to verify that the $\kappa$-connection (51) does produce the $\kappa$-dS composition law when applying the geometric construction of the composition law encoded in Eqs. (13)-(15).

We start by noticing that the equation 13 , which defines the composition law associated to a given affine connection, in the case of the $\kappa$-connection takes the form:

$$
\left\{\begin{array}{l}
\frac{d}{d t} \frac{d}{d s} \gamma_{0}(s, t)=0 \\
\frac{d}{d t} \frac{d}{d s} \gamma_{1}(s, t)+\ell \frac{d \zeta_{0}(s, t)}{d t} \frac{d \gamma_{1}(s, t)}{d s}=0
\end{array}\right.
$$

where $\zeta$ is the connection geodesic connecting $\gamma(s, 0)$ to $\gamma(s, 1)$, which means

$$
\left\{\begin{array}{l}
\frac{d^{2}}{d t^{2}} \zeta_{0}(s, t)=0 \\
\frac{d^{2}}{d t^{2}} \zeta_{1}(s, t)+\ell \frac{d \zeta_{0}(s, t)}{d t} \frac{d \zeta_{1}(s, t)}{d t}=0 \\
\zeta_{1}(s, 0)=\gamma(s, 0) \\
\zeta_{1}(s, 1)=\gamma(s, 1)
\end{array}\right.
$$

The solution for $\zeta$ is easily found to take the form

$$
\left\{\begin{array}{l}
\zeta_{0}(s, t)=\gamma_{0}(s, 0)+\Delta_{0} t \\
\zeta_{1}(s, t)=\gamma_{1}(s, 0)+\Delta_{1} \frac{\left(1-e^{-\ell \Delta_{0} t}\right)}{\left(1-e^{-\ell \Delta_{0}}\right)}
\end{array}\right.
$$


where

$$
\Delta_{\lambda}=\gamma_{\lambda}(s, 1)-\gamma_{\lambda}(s, 0)
$$

Using this solution one then finds that $\gamma$ must satisfy

$$
\left\{\begin{array}{l}
\frac{d}{d t} \frac{d}{d s} \gamma_{0}(s, t)=0 \\
\frac{d}{d t} \frac{d}{d s} \gamma_{1}(s, t)+\ell \Delta_{0} \frac{d \gamma_{1}(s, t)}{d s}=0
\end{array}\right.
$$

In turn these, once the boundary conditions $(12)$ are imposed on $\gamma(s, t)$, lead to the results

$$
\left\{\begin{array}{l}
\gamma_{0}(s, t)=\gamma_{0}^{(k)}(s)+\gamma_{0}^{(q)}(t) \\
\gamma_{1}(s, t)=\gamma_{1}^{(q)}(t)+\gamma_{1}^{(k)}(s) e^{-\ell q_{0} t}
\end{array}\right.
$$

where $\gamma^{(q)}\left(\gamma^{(k)}\right)$ is the connection geodesic connecting the point $q$ (the point $k$ ) to the origin.

Then, using our formulation of the composition law given in terms of $q \oplus_{\ell} k=\gamma(1,1)$, one easily finds that

$$
\left\{\begin{array}{l}
\left(q \oplus_{\ell} k\right)_{0}=q_{0}+k_{0} \\
\left(q \oplus_{\ell} k\right)_{1}=q_{1}+k_{1} e^{-\ell q_{0}}
\end{array}\right.
$$

which indeed successfully reproduces the $\kappa$-dS composition law.

Following steps completely analogous to the ones discussed in this subsection it is easy to verify that one obtains again correctly the $\kappa$-dS composition law also using the alternative definition for the affine connection given in 21 (and of course then using the corresponding steps of derivation, summarized in the previous section in Eqs. (22)).

\section{B. Two $\kappa$-dS geometries}

The last point we want to make in this section on the $\kappa$-dS momentum space concerns again the ambiguity we highlighted for what concerns the association of an affine connection to a given law of composition of momenta. We recall here that the ambiguity is connected with the choice between the following two options:

$$
\left\{\begin{array}{l}
\Gamma_{(1) \nu}^{\lambda \mu}(p)=-\left.\frac{\partial}{\partial q_{\lambda}} \frac{\partial}{\partial k_{\mu}}\left(q \oplus_{\ell}^{[p]} k\right)_{\nu}\right|_{q=k=p} \\
\Gamma_{(2)^{\nu}}^{\lambda \mu}(p)=-\left.\frac{\partial}{\partial k_{\lambda}} \frac{\partial}{\partial q_{\mu}}\left(q \oplus_{\ell}^{[p]} k\right)_{\nu}\right|_{q=k=p}
\end{array}\right.
$$

As stressed at the end of the previous subsection, in the $\kappa$-dS case one can explicitly verify that both of these possibilities for the affine connection give us back the $\kappa$-dS composition law. This confirms that the choice between $\Gamma_{(1)}$ and $\Gamma_{(2)}$ is merely conventional. But it is still interesting to assess how this ambiguity affects the geometric structure of $\kappa$-dS momentum space. As a step toward doing this let us start by recalling that (as reviewed in the appendix A, given a metric, any affine connection can be split in a unique way as follows:

$$
\Gamma_{\nu}^{\lambda \mu}=A_{\nu}^{\lambda \mu}+K_{\nu}^{\lambda \mu}+V_{\nu}^{\lambda \mu}
$$

where $K$ is the contortion tensor, defined by

$$
K_{\rho}^{\lambda \mu} g^{\rho \nu}+K_{\rho}^{\lambda \nu} g^{\rho \mu}=0
$$

and $V$ is the cononmetricity, defined by

$$
\nabla_{(\Gamma)}^{\lambda} g^{\mu \nu}=V_{\rho}^{\lambda \mu} g^{\rho \nu}+V_{\rho}^{\lambda \nu} g^{\rho \mu}
$$

Next let us observe that for the $\kappa$-dS composition law 45 one finds that

$$
\left\{\begin{array}{l}
\Gamma_{(1) \nu}^{\lambda \mu}=\ell \delta_{0}^{\lambda} \delta_{1}^{\mu} \delta_{\nu}^{1} \\
\Gamma_{(2)^{\nu}}^{\lambda \mu}=\ell \delta_{0}^{\mu} \delta_{1}^{\lambda} \delta_{\nu}^{1}
\end{array}\right.
$$

while the Levi-Civita connection on de Sitter momentum space is

$$
A_{\nu}^{\lambda \mu}=\ell\left(\delta_{0}^{\lambda} \delta_{1}^{\mu}+\delta_{1}^{\lambda} \delta_{0}^{\mu}\right) \delta_{\nu}^{1}+\ell e^{2 \ell p_{0}} \delta_{1}^{\lambda} \delta_{1}^{\mu} \delta_{\nu}^{0}
$$


By subtracting this $A^{\lambda \mu}{ }_{\nu}$ to both $\Gamma_{(1)}$ and $\Gamma_{(2)}$ we can get the contortion and the cononmetricity in the two cases:

$$
\begin{cases}K_{(1) \lambda}^{\mu \nu} \lambda=-\ell \delta_{1}^{\mu}\left(\delta_{1}^{\nu} \delta_{\lambda}^{0} e^{2 \ell p_{0}}+\delta_{\nu}^{0} \delta_{\lambda}^{1}\right) & K_{(2) \lambda}^{\mu \nu}=-K_{(1) \lambda}^{\mu \nu} \lambda \\ V_{(1) \lambda}^{\mu \nu}=0 & V_{(2) \lambda}^{\mu \nu} \lambda=2 K_{(1) \lambda}^{\mu \nu} \lambda\end{cases}
$$

Notice in particular that $\Gamma_{(1)}$ has no cononmetricity whereas for $\Gamma_{(2)}$ the cononmetricity does not vanish. This illustrates the issue we raised above: results on the relative-locality framework with the $\kappa$-dS momentum space cannot be used for developing any general intuition on the role of cononmetricity in this sort of theories, since the $\kappa$-dS theory can be viewed in equally legitimate manner both as a case of momentum space with cononmetricity and as a case of momentum space without cononmetricity.

\section{V. "PROPER" DE SITTER MOMENTUM SPACE}

The $\kappa$-dS momentum space has been a preferred choice for the first studies done within the relative-locality framework also for the simplifications afforded by the $\kappa$-connection and corresponding simple properties of the composition law, which in particular is associative. The "price to pay" for that associativity is the noncommutativity of the composition law on the $\kappa$-momentum space. One of the main objectives of the study we are here reporting is to propose a momentum space which could be the most natural starting point for the first investigations of the relative-locality framework when the composition law is not associative but it is commutative. The case we propose for these purposes is "proper" de Sitter momentum space (which we label alternatively as the proper-dS momentum space or simply as the dS momentum space). By this we mean a momentum space whose metric is a de Sitter metric, like in the $\kappa$-dS case, and as the affine connection on momentum space one takes the Levi-Civita connection of the de Sitter metric (rather than the $\kappa$-connection). So we deal with the same metric as in the previous section,

$$
g^{\mu \nu}(p)=\left(\begin{array}{cc}
1 & 0 \\
0 & -e^{2 \ell p_{0}}
\end{array}\right)
$$

but now studied in combination with its associated Levi-Civita connection

$$
A_{\nu}^{\lambda \mu}=\ell\left(\delta_{0}^{\lambda} \delta_{1}^{\mu}+\delta_{0}^{\mu} \delta_{1}^{\lambda}\right) \delta_{\nu}^{1}+\ell e^{2 \ell p_{0}} \delta_{1}^{\lambda} \delta_{1}^{\mu} \delta_{\nu}^{0}
$$

rather than the $\kappa$-connection.

Since the on-shell relation is determined exclusively by the metric, for theories on this proper-dS momentum space one must enforce the same on-shellness as in the $\kappa$-dS case:

$$
\operatorname{Cosh}[\ell m]=\operatorname{Cosh}\left[\ell d_{\ell}(p)\right]=\operatorname{Cosh}\left[\ell p_{0}\right]-\frac{\ell^{2}}{2} p_{1}^{2} e^{\ell p_{0}}
$$

We find that this setup leads to a commutative (but non-associative) composition law when assuming for the link between composition law and affine connection the novel geometric-interpretation prescription we introduced in Eqs. 13 - 15 .

Contrary to the $\kappa$-momentum case, in this case of combining the de Sitter metric with its Levi-Civita connection one does find some differences between the standard geometric-interpretation prescription, here coded in Eq.(6), and the novel geometric interpretation we introduced through Eqs. (13)-(15). When the analysis is done with the prescription (6) the composition law associated to the Levi-Civita connection is not necessarily commutative, but, in a sense that we shall soon clarify, one can choose to make it commutative. Even when that particular choice of commutativity of the composition law is made the resulting composition law is different from the one obtained from the novel geometric-interpretation prescription we introduced in Eqs. (13)- 1 (15).

\section{A. Weakly-proper dS}

One of our main goals is to find a natural candidate for a commutative composition law on a momentum space with dS metric, and our preferred scenario when this project got started was to find that such a picture could be directly linked to the adoption of the Levi-Civita connection on dS momentum space. In the next subsection we shall report a picture which exactly matches these desiderata and expectations, relying on the novel geometricinterpretation prescription we introduced in Eqs. (13)-(15). We shall label that picture as "proper dS momentum 
space". Before getting to those results, in this subsection we show that also adopting the standard geometricinterpretation prescription, here coded in Eq.(6), one can have on dS momentum space a commutative composition law paired with the Levi-Civita connection, but the logical link between these two structures is weaker, in the sense already here clarified in Sec.III (and visible again in the points we make in this subsection). We shall then label the picture arising in this subsection as the "weakly-proper dS momentum space".

Our road to this weakly-proper dS momentum space starts of course again from (6):

$$
\Gamma_{\lambda}^{\mu \nu}(p)=-\left.\frac{\partial}{\partial q_{\mu}} \frac{\partial}{\partial k_{\nu}}\left(q \oplus_{\ell}^{[p]} k\right)_{\lambda}\right|_{q=k=p}
$$

Using notation and results from Sec.III, now specialized to the case of the Levi-Civita connection on dS momentum space, (71), one easily finds that

$$
X_{\lambda}^{\mu \nu}=\left(\delta_{0}^{\lambda} \delta_{1}^{\mu}+\delta_{0}^{\mu} \delta_{1}^{\lambda}\right) \delta_{\nu}^{1}+\delta_{1}^{\lambda} \delta_{1}^{\mu} \delta_{\nu}^{0}
$$

and

$$
Z_{\lambda}^{\alpha \mu \nu}=\ell^{2}\left[\delta_{0}^{\mu} \delta_{\lambda}^{1} \delta_{0}^{\nu} \delta_{1}^{\alpha}+\frac{1}{2} \delta_{1}^{\mu} \delta_{\lambda}^{1} \delta_{1}^{\alpha} \delta_{1}^{\nu}\right]
$$

So the family of composition laws that can be associated to the de Sitter Levi-Civita connection on dS momentum space according to the standard geometric-interpretation prescription, here coded in Eq. (6), takes the form:

$$
\left(q \oplus_{\ell} k\right)_{\lambda}=q_{\lambda}+k_{\lambda}-A_{\lambda}^{\alpha \beta}(0) q_{\alpha} k_{\beta}+\ell^{2} Y_{\lambda}^{\alpha \beta \gamma} q_{\alpha} q_{\beta} k_{\gamma}+\ell^{2} \delta_{\lambda}^{1}\left(q_{1} k_{0}^{2}+\frac{1}{2} q_{1} k_{1}^{2}\right) q_{\alpha} k_{\mu} k_{\nu}
$$

where $A_{\lambda}^{\alpha \beta}$ is the de Sitter Levi-Civita connection, whose form was noted in 71 , and $Y_{\lambda}^{\alpha \beta \gamma}$ is an arbitrary constant tensor. Eq. 76 can be rewritten more explicitly as follows

$$
\left\{\begin{array}{l}
\left(q \oplus_{\ell} k\right)_{0}=q_{0}+k_{0}-\ell q_{1} k_{1}+\ell^{2} Y_{0}^{\alpha \beta \gamma} q_{\alpha} q_{\beta} k_{\gamma} \\
\left(q \oplus_{\ell} k\right)_{1}=q_{1}+k_{1}-\ell\left(q_{0} k_{1}+q_{1} k_{0}\right)+\ell^{2} Y_{1}^{\alpha \beta \gamma} q_{\alpha} q_{\beta} k_{\gamma}+\ell^{2}\left(q_{1} k_{0}^{2}+\frac{1}{2} q_{1} k_{1}^{2}\right)
\end{array}\right.
$$

Evidently this family of composition laws includes a possibility which is commutative, and is therefore of particular interest for some of the objectives of our analysis. This commutative composition law is

$$
\left\{\begin{array}{l}
\left(q \oplus_{\ell} k\right)_{0}=q_{0}+k_{0}-\ell q_{1} k_{1} \\
\left(q \oplus_{\ell} k\right)_{1}=q_{1}+k_{1}-\ell\left(q_{0} k_{1}+q_{1} k_{0}\right)+\ell^{2}\left(q_{1} k_{0}^{2}+q_{0}^{2} k_{1}+\frac{1}{2} q_{1} k_{1}^{2}+\frac{1}{2} q_{1}^{2} k_{1}\right)
\end{array}\right.
$$

We shall label as weakly-proper dS momentum space the case characterized (to quadratic order in $\ell$ ) by this commutative composition law and the on-shellness determined by the dS metric on momentum space.

We observe that the composition law (78) is not associative,

$$
\left(\left(q \oplus_{\ell} k\right) \oplus_{\ell} p\right)_{\lambda} \neq\left(q \oplus_{\ell}\left(k \oplus_{\ell} p\right)\right)_{\lambda}
$$

and one easily verifies that it does not satisfy neither the left loop rule nor the right loop rule:

$$
\begin{aligned}
& q \oplus_{\ell}\left(\ominus_{\ell} q \oplus_{\ell} p\right) \neq p \\
& \left(p \oplus_{\ell} \ominus_{\ell} q\right) \oplus_{\ell} q \neq p .
\end{aligned}
$$

\section{B. Proper dS}

Our next task is to show that the novel geometric-interpretation prescription we introduced in Eqs. 13)-15 associates the Levi-Civita connection on momentum space to a specific commutative composition law.

We show in appendix $\mathrm{C}$ how to derive from our Eqs. (13)-(15) a second-order-in- $\ell$ expression for the composition law. This requires expanding the connection as follows:

$$
\Gamma_{\nu}^{\lambda \mu}(\zeta)=\Gamma_{\nu}^{\lambda \mu}(0)+\partial^{\rho} \Gamma_{\nu}^{\lambda \mu}(0) \zeta_{\rho}^{(0)}
$$


where we defined $\zeta^{(0)}$ as the zero-th order approximation of $\zeta$ (definitions in Eqs.13)-15).

Adopting analogous expansions for $\gamma$ and $\zeta$ one then obtains with simple steps of derivation the result

$$
\left(q \oplus_{\ell} k\right)_{\lambda}=q_{\lambda}+k_{\lambda}-\ell \bar{\Gamma}_{\lambda}^{\alpha \beta} q_{\alpha} k_{\beta}-\ell^{2}\left(\partial^{\rho} \bar{\Gamma}_{\lambda}^{\alpha \beta}\right) q_{\alpha} k_{\beta}\left(\frac{q_{\rho}+k_{\rho}}{2}\right)+\frac{1}{2} \ell^{2} \bar{\Gamma}_{\lambda}^{\alpha \beta} \bar{\Gamma}_{\alpha}^{\gamma \delta} q_{\gamma} k_{\delta} k_{\beta}+\frac{1}{2} \ell^{2} \bar{\Gamma}_{\lambda}^{\alpha \beta} \bar{\Gamma}_{\beta}^{\gamma \delta} q_{\alpha} q_{\gamma} k_{\delta}
$$

where again we use the notation $\ell \bar{\Gamma}^{\mu \nu}{ }_{\lambda} \equiv \Gamma_{\lambda}^{\mu \nu}(0),\left.\ell^{2} \partial^{\theta} \bar{\Gamma}_{\lambda}^{\mu \nu} \equiv \partial^{\theta} \Gamma_{\lambda}^{\mu \nu}(p)\right|_{p=0}$. Substituting in this result the explicit form of the Levi-Civita connection (71) one then finally obtains

$$
\left\{\begin{array}{l}
\left(q \oplus_{\ell} k\right)_{0}=q_{0}+k_{0}-\ell q_{1} k_{1}+\frac{\ell^{2}}{2}\left[-q_{1} k_{1}\left(q_{0}+k_{0}\right)+q_{0} k_{1}^{2}+q_{1}^{2} k_{0}\right] \\
\left(q \oplus_{\ell} k\right)_{1}=q_{1}+k_{1}-\ell\left(q_{0} k_{1}+q_{1} k_{0}\right)+\frac{\ell^{2}}{2}\left[\left(q_{0} k_{1}+q_{1} k_{0}\right)\left(q_{0}+k_{0}\right)+q_{1} k_{1}^{2}+q_{1}^{2} k_{1}\right]
\end{array}\right.
$$

Our proper-dS momentum space is characterized (to quadratic order in $\ell$ ) by this commutative composition law and the on-shellness determined by the dS metric on momentum space.

The composition law (84) is not associative. We opt to show this by first deriving some more general results. Specifically we observe that when composition laws are derived from the momentum-space affine connection using our prescription, so that 83 holds, the associativity (or lack thereof) of the composition law is linked to the connection, at second order in $\ell$, through the relationship

$$
\left(\left(q \oplus_{\ell} k\right) \oplus_{\ell} p\right)_{\lambda}-\left(q \oplus_{\ell}\left(k \oplus_{\ell} p\right)\right)_{\lambda}=-\frac{\ell^{2}}{2}\left(\bar{F}_{\lambda}^{\rho \beta \alpha}+\nabla_{\Gamma}^{\rho} \bar{T}_{\lambda}^{\alpha \beta}\right) k_{\alpha} p_{\beta} q_{\rho}
$$

where $\ell^{2} \nabla_{\Gamma} \bar{T}$ and $\ell^{2} \bar{F}$ are, respectively, the covariant derivative of the torsion contribution to the connection and the curvature of the connection, so that in particular

$$
\bar{F}_{\lambda}^{\rho \beta \alpha}=\partial^{\rho} \bar{\Gamma}_{\lambda}^{\beta \alpha}-\partial^{\beta} \bar{\Gamma}_{\lambda}^{\rho \alpha}+\bar{\Gamma}_{\lambda}^{\rho \tau} \bar{\Gamma}_{\tau}^{\beta \alpha}-\bar{\Gamma}_{\lambda}^{\beta \tau}{ }_{\lambda} \bar{\Gamma}_{\tau}^{\rho \alpha}
$$

Again in 85 and 86 it is intended that the fields are to be evaluated in the origin of momentum space.

For the specific case of our proper-dS momentum space we evidently have no torsion and the curvature of the connection is, to all orders,

$$
F_{\lambda}^{\rho \beta \alpha}(p)=2 \ell^{2} \delta_{0}^{[\rho} \delta_{1}^{\beta]}\left(e^{2 \ell p_{0}} \delta_{1}^{\alpha} \delta_{\lambda}^{0}+\delta_{\lambda}^{1} \delta_{0}^{\alpha}\right)
$$

which translates into a non-associativity of the form

$$
\left(\left(q \oplus_{\ell} k\right) \oplus_{\ell} p\right)_{\lambda}-\left(q \oplus_{\ell}\left(k \oplus_{\ell} p\right)\right)_{\lambda}=\frac{\ell^{2}}{2}\left(\delta_{\lambda}^{0} k_{1}+\delta_{\lambda}^{1} k_{0}\right)\left(p_{0} q_{1}-p_{1} q_{0}\right)
$$

We also notice in closing that for the proper-dS composition law 84 both the left loop inverse rule and the right loop inverse rule are not verified.

\section{DSR-RELATIVISTIC PROPERTIES OF PROPER-DS AND $\kappa$-DS MOMENTUM SPACES}

As stressed already in Sec.I we are not just interested in dS momentum spaces with commutative composition laws: we are also looking for rules of kinematics which are relativistic. Since special-relativistic laws of transformation cannot be symmetries of any de Sitter momentum space, relativistic invariance must be inevitably described within the structure of "DSR relativistic theories" [4] (also see Refs. 20 25]), theories with two relativistic invariants, the speed-of-light scale $c$ and a length/inverse-momentum scale: the scale $\ell$ that characterizes the geometry of momentum space must in fact be an invariant if the theories on such momentum spaces are to be relativistic.

As already noticed in Ref. [18] the requirement that a curved momentum space be "DSR compatible" is strongly constraining: with arbitrary combinations of a metric and of an affine connection one in general ends up with a combination of law of on-shellness and law of energy-momentum conservation which allows the identification of a preferred frame. But enforcing a suitable compatibility between metric and affine connection one does find relativistic momentum spaces [18, and we shall now show that all 3 cases we considered, the $\kappa$-dS case, the proper-dS case and the weakly-proper-dS case, are indeed DSR relativistic. 


\section{A. The starting point of the relativistic properties of Minkowski momentum space}

We set the stage for our analysis of relativistic properties of $1+1$-dimensional $\kappa$-dS and "proper-dS" momentum spaces by first reviewing a few known facts about the special-relativistic properties of the standard Minkowski momentum space.

For Minkowski momentum space of course the on-shellness has the form

$$
m^{2}(p)=E^{2}-p^{2}=\eta^{\mu \nu} p_{\mu} p_{\nu}
$$

and the conservation of energy-momentum has the form

$$
p_{\mu}=q_{\mu}+k_{\mu}
$$

(focusing again on the example of a two-body particle decay).

The relativistic compatibility of Minkowski momentum space is ensured by the standard special-relativistic rules of transformation of momenta, which in particular for boosts we shall characterize in terms of

$$
p_{\mu} \rightarrow \Lambda_{\mu}^{\xi}(p)
$$

where indeed we denoted by $\Lambda^{\xi}$ a standard Lorentz boost of rapidity/boost parameter $\xi$, with generator $N$ such that (for small $\xi$ )

$$
p_{\mu} \rightarrow \Lambda_{\mu}^{\xi}(p) \approx p_{\mu}+\xi N_{\mu}(p)
$$

Indeed under these standard boosts one easily finds that the on-shellness is observer independent,

$$
m^{2}\left(\Lambda^{\xi}(p)\right)=m^{2}(p),
$$

and also the composition law is observer independent,

$$
p_{\mu}=q_{\mu}+k_{\mu} \rightarrow \Lambda_{\mu}^{\xi}(p)=\Lambda_{\mu}^{\xi}(q)+\Lambda_{\mu}^{\xi}(k) .
$$

We also note briefly that in special relativity the on-shellness is also invariant under momentum-space translations. To see in which sense this holds let us consider two arbitrary points on the Minkowskian momentum space, $k$ and $q$, and their distance

$$
d^{2}(k, q)=\eta^{\mu \nu}\left(k_{\mu}-q_{\mu}\right)\left(k_{\mu}-q_{\mu}\right)
$$

This is not only invariant under boosts but also under the full Poincare group, with momentum-space translations of course given by

$$
q_{\mu} \rightarrow q_{\mu}+a_{\mu}
$$

\section{B. DSR-relativistic compatibility of "proper dS" momentum space}

Let us now start considering the case of our "proper-dS" momentum space. As we shall see this is a case where the new laws of kinematics

$$
\left\{\begin{array}{l}
m^{2}=d_{\ell}^{2}(p) \\
p_{\mu}=\left(q \oplus_{\ell} k\right)_{\mu}
\end{array}\right.
$$

are still compatible with a rather standard implementation of observer independence, involving of course DSRdeformed boost transformations $\tilde{\Lambda}$. As we shall soon show, the action of these deformed boosts on a momentum $p_{\mu}$ is governed by

$$
B^{\xi} \triangleright p_{\mu}=\tilde{\Lambda}_{\mu}^{\xi}(p) \approx p_{\mu}+\xi \tilde{N}_{\mu}(p)
$$

with

$$
\left\{\begin{array}{l}
\tilde{N}_{0}(p)=p_{1} \\
\tilde{N}_{1}(p)=\frac{1}{2 \ell}\left(1-e^{-2 \ell p_{0}}\right)-\frac{\ell}{2} p_{1}^{2}
\end{array}\right.
$$


It is easy to check (see below) that the condition of on-shellness is observer independent under these transformation laws

$$
d_{\ell}^{2}\left(B^{\xi} \triangleright p\right)=d_{\ell}^{2}(p)=m_{p}^{2}
$$

It is already well established [4, 18, 24, 25] that the full (DSR-)relativistic compatibility of a momentum space also imposes strong demands on the action of boosts on composed momenta: $B_{\xi} \triangleright\left(a \oplus_{\ell} b\right)$. This is the part that is most challenging from a relativistic perspective and it shall prove to be particularly striking when we get to the point of analyzing the relativistic properties of the $\kappa$-momentum space. For the case of our proper-dS momentum space it turns out that the action of boosts on composed momenta retains most of its intuitive properties, and in particular is such that

$$
B^{\xi} \triangleright\left(q \oplus_{\ell} k\right)=\tilde{\Lambda}^{\xi}(q) \oplus_{\ell} \tilde{\Lambda}^{\xi}(k)
$$

with

$$
B^{\xi} \triangleright\left(q \oplus_{\ell} k\right)=\left.\tilde{\Lambda}_{\mu}^{\xi}(\mathcal{P})\right|_{\mathcal{P}=q \oplus_{\ell} k}
$$

All this can be summarized, in the spirit and notation of Ref. [18, in terms of the following intuitive property of boost generators:

$$
\tilde{N}_{\left[q \oplus_{\ell} k\right]}^{\xi}=\tilde{N}_{[q]}^{\xi}+\tilde{N}_{[k]}^{\xi}
$$

meaning that the action of the boost generator on the composed momenta is simply obtained by acting on each momentum in the composition, following a standard implementation of Leibniz rule.

We shall provide support for these claims while observing incidentally that also for our "proper dS" momentum space (just like stressed at the end of the previous subsection for the standard case of Minkowskian momentum space) the distance between points of momentum space is invariant not only under boosts but also under some appropriate notion of momentum-space translations. So we look for transformations $\mathcal{S}$ that leave invariant the distance on momentum space:

$$
d_{\ell}^{2}(\mathcal{S}(k), \mathcal{S}(q))=d_{\ell}^{2}(k, q)
$$

where $\mathcal{S}$ must be regarded as a particular class of diffeomorphisms on momentum space:

$$
\mathcal{S}: p_{\mu} \rightarrow \mathcal{S}_{\mu}(p)
$$

which solves (104) and reduces to the undeformed Poincaré group of transformations in the $\ell \rightarrow 0$ limit.

We focus on the infinitesimal set of diffeomorphisms:

$$
\mathcal{S}_{\mu}(p) \approx p_{\mu}+\mathcal{T}_{\mu}(p)
$$

where the transformations $\mathcal{T}$ can be considered as the generators of the deformed Poincare group. In terms of the generators $\mathcal{T}$ then the request of invariance of the distance on momentum space takes the form:

$$
d_{\ell}^{2}(k+\mathcal{T}(k), q+\mathcal{T}(q))=d_{\ell}^{2}(k, q)
$$

which we are going to solve at first order in $\mathcal{T}$.

By defining $L(\gamma, \dot{\gamma})=\sqrt{g^{\mu \nu}(\gamma) \dot{\gamma}_{\mu} \dot{\gamma}_{\nu}}$ and using the definition of $d_{\ell}^{2}$ one easily finds

$$
0=\delta \int d t L(\gamma(t), \dot{\gamma}(t))=\int\left(\frac{\partial L}{\partial \gamma}-\frac{d}{d t}\left(\frac{\partial L}{\partial \dot{\gamma}}\right)\right) \delta \gamma d t+\left.\left(\frac{\partial L}{\partial \dot{\gamma}} \delta \gamma\right)\right|_{t=0} ^{t=1}
$$

where $\gamma$ is the curve connecting $q$ to $k$ and $\delta \gamma(t)$ is the variation of $\gamma(t)$ due to the variation of its extremal points $q$ and $k$, which must satisfy $\delta \gamma(0)=\mathcal{T}(q)$ and $\delta \gamma(1)=\mathcal{T}(k)$.

Using the definition of $L$ the integrand term on the right-hand side of $(108)$ is easily seen to be the geodesic equation which is solved, by definition, by the curve $\gamma$. We are then left with the boundary term:

$$
\left.\frac{d}{d t}\left(\frac{\partial L}{\partial \dot{\gamma}} \delta \gamma\right)\right|_{t=0} ^{t=1}=0
$$


We show in appendix $(\mathrm{B})$ that, enforcing the validity of the previous equation for any couple of points $q$ and $k$, translates in a requirement for the generators $\mathcal{T}$ of the form

$$
\nabla_{(A)}^{(\mu} \mathcal{T}^{\nu)}=0
$$

where $\nabla_{A}$ is the covariant derivative associated to the Levi-Civita connection $A^{\lambda \mu}{ }_{\nu}$ defined by the momentum-space metric $g^{\mu \nu}$. Evidently we found, with (110), that $\mathcal{T}$ must satisfy a killing equation. And this killing equation admits solution for three classes of metrics, the ones that are diffeomorphic to Minkowski, de Sitter or anti de Sitter metric. We are here focusing for definiteness on the de Sitter case.

For the de Sitter momentum space, still working in $1+1$ dimension, it is easy to show, as we do in appendix $B$ that the solutions of 110 are:

$$
\left\{\begin{array}{l}
\mathcal{T}_{0}(p)=\xi p_{1}+\gamma \\
\mathcal{T}_{1}(p)=\xi\left(\frac{1}{2 \ell}\left(1-e^{-2 \ell p_{0}}\right)-\frac{\ell}{2} p_{1}^{2}\right)-\ell p_{1} \gamma+\beta
\end{array}\right.
$$

Here, $\xi, \beta$ and $\gamma$ arise as constants of integration of the killing equation, and have to be taken as the infinitesimal parameters associated to the boost and (two) translations in the de Sitter momentum space.

The $\ell$-deformed boost generators, which are of our primary interest, can be easily read off (111):

$$
\left\{\begin{array}{l}
\tilde{N}_{0}(p)=p_{1} \\
\tilde{N}_{1}(p)=\left(\frac{1}{2 \ell}\left(1-e^{-2 \ell p_{0}}\right)-\frac{\ell}{2} p_{1}^{2}\right)
\end{array}\right.
$$

which, confirming our earlier claims, coincide with 99 .

This choice of deformed boost transformations ensures by construction the observer independence of the on-shell relation. Our next task is to show that it also ensures the observer independence of energy-momentum conservation laws, which we shall investigate at the level of the properties of the the law $\oplus_{\ell}$ of composition of momenta. In this subsection we shall be satisfied performing this analysis at second order in $\ell$. We start by noticing that at second order in $\ell$ the boost generator 99 takes the form

$$
\left\{\begin{array}{l}
\tilde{N}_{0}(p) \simeq p_{1} \\
\tilde{N}_{1}(p) \simeq\left(p_{0}-\ell p_{0}^{2}-\frac{\ell}{2} p_{1}^{2}+\frac{1}{3} \ell^{2} p_{0}^{3}\right)
\end{array}\right.
$$

The announced DSR-relativistic compatibility of our proper-dS momentum space, taking the form of (101)-(102) is automatically ensured by verifying that (at leading order in the boost parameter $\xi$ )

$$
\left.\tilde{N}_{\mu}(\mathcal{P})\right|_{\mathcal{P}=q \oplus_{\ell} k}=\frac{\partial}{\partial q_{\rho}}\left(q \oplus_{\ell} k\right)_{\mu} \tilde{N}_{\rho}(q)+\frac{\partial}{\partial k_{\rho}}\left(q \oplus_{\ell} k\right)_{\mu} \tilde{N}_{\rho}(k)
$$

The validity of this 114 is easily checked by computing separately its two sides and finding that they give the same result. It suffices to substitute the explicit form of the second order boost 113 and of the second order proper-dS composition law computed in (84), so that one gets for the left-hand side of (114)

$$
\left.\tilde{N}_{0}(\mathcal{P})\right|_{\mathcal{P}=q \oplus \ell k}=q_{1}+k_{1}-\ell\left(q_{0} k_{1}+q_{1} k_{0}\right)+\frac{\ell^{2}}{2}\left[\left(q_{0} k_{1}+q_{1} k_{0}\right)\left(q_{0}+k_{0}\right)+q_{1} k_{1}^{2}+q_{1}^{2} k_{1}\right]
$$

and

$$
\left.\tilde{N}_{1}(\mathcal{P})\right|_{\mathcal{P}=q \oplus_{\ell} k}=q_{0}+k_{0}-l\left[q_{1} k_{1}+\left(q_{0}+k_{0}\right)^{2}+\frac{1}{2}\left(q_{1}+k_{1}\right)^{2}\right]+l^{2}\left(\frac{5}{2} q_{0} q_{1} k_{1}+\frac{5}{2} k_{0} q_{1} k_{1}+\frac{3}{2} q_{1}^{2} k_{0}+\frac{3}{2} q_{0} k_{1}^{2}\right)+\frac{2}{3} l^{2}(q+k)_{0}^{3}
$$

while for the right-hand side of 114 one gets

$$
\begin{gathered}
\frac{\partial}{\partial q_{\rho}}\left(\left(q \oplus_{\ell} k\right)_{0}\right) \tilde{N}_{\rho}(q)+\frac{\partial}{\partial k_{\rho}}\left(\left(q \oplus_{\ell} k\right)_{0}\right) \tilde{N}_{\rho}(k)= \\
=\frac{\partial}{\partial q_{\rho}}\left(q_{0}+k_{0}-\ell q_{1} k_{1}+\frac{\ell^{2}}{2}\left[-q_{1} k_{1}\left(q_{0}+k_{0}\right)+q_{0} k_{1}^{2}+q_{1}^{2} k_{0}\right]\right) \tilde{N}_{\rho}(q)+ \\
+\frac{\partial}{\partial k_{\rho}}\left(q_{0}+k_{0}-\ell q_{1} k_{1}+\frac{\ell^{2}}{2}\left[-q_{1} k_{1}\left(q_{0}+k_{0}\right)+q_{0} k_{1}^{2}+q_{1}^{2} k_{0}\right]\right) \tilde{N}_{\rho}(k)= \\
=q_{1}+k_{1}-l\left(k_{1} q_{0}+q_{1} k_{0}\right)+\frac{l^{2}}{2}\left[k_{1} q_{0}^{2}+k_{1} q_{1}^{2}+q_{1} k_{0}^{2}+q_{1} k_{1}^{2}+q_{0} k_{1} k_{0}+q_{1} q_{0} k_{0}\right]
\end{gathered}
$$


and

$$
\begin{gathered}
\frac{\partial}{\partial q_{\rho}}\left(\left(q \oplus_{\ell} k\right)_{1}\right) \tilde{N}_{\rho}(q)+\frac{\partial}{\partial k_{\rho}}\left(\left(q \oplus_{\ell} k\right)_{1}\right) \tilde{N}_{\rho}(k)= \\
=\frac{\partial}{\partial q_{\rho}}\left(q_{1}+k_{1}-\ell\left(q_{0} k_{1}+q_{1} k_{0}\right)+\frac{\ell^{2}}{2}\left[\left(q_{0} k_{1}+q_{1} k_{0}\right)\left(q_{0}+k_{0}\right)+q_{1} k_{1}^{2}+q_{1}^{2} k_{1}\right]\right) \tilde{N}_{\rho}(q)+ \\
+\frac{\partial}{\partial k_{\rho}}\left(q_{1}+k_{1}-\ell\left(q_{0} k_{1}+q_{1} k_{0}\right)+\frac{\ell^{2}}{2}\left[\left(q_{0} k_{1}+q_{1} k_{0}\right)\left(q_{0}+k_{0}\right)+q_{1} k_{1}^{2}+q_{1}^{2} k_{1}\right]\right) \tilde{N}_{\rho}(k)= \\
=q_{0}+k_{0}-l\left(q_{1} k_{1}+\left(q_{0}+k_{0}\right)^{2}+\frac{1}{2}\left(q_{1}+k_{1}\right)^{2}\right)+l^{2}\left(\frac{5}{2} q_{0} q_{1} k_{1}+\frac{5}{2} k_{0} q_{1} k_{1}+\frac{3}{2} q_{1}^{2} k_{0}+\frac{3}{2} q_{0} k_{1}^{2}\right)+\frac{2}{3} l^{2}(q+k)_{0}^{3}
\end{gathered}
$$

This completes our verification of the (DSR-)relativistic compatibility of the "proper dS" momentum space to second order in $\ell$.

We stress that by checking the observer independence of the law of composition of two momenta we automatically also ensured the observer independence of the composition of any number of momenta. For example, one easily sees that

$$
\left.\tilde{\Lambda}_{\mu}^{\xi}(\mathcal{P})\right|_{\mathcal{P}=a \oplus_{\ell} b}=\left.\tilde{\Lambda}_{\mu}^{\xi}(a) \oplus_{\ell} \tilde{\Lambda}_{\mu}^{\xi}(b) \Longrightarrow \tilde{\Lambda}_{\mu}^{\xi}(\mathcal{P})\right|_{\mathcal{P}=a \oplus_{\ell} b \oplus_{\ell} c}=\left.\tilde{\Lambda}_{\mu}^{\xi}\left(\mathcal{P}^{\prime}\right)\right|_{\mathcal{P}^{\prime}=a \oplus_{\ell} b} \oplus_{\ell} \tilde{\Lambda}_{\mu}^{\xi}(c)=\tilde{\Lambda}_{\mu}^{\xi}(a) \oplus_{\ell} \tilde{\Lambda}_{\mu}^{\xi}(b) \oplus_{\ell} \tilde{\Lambda}_{\mu}^{\xi}(c)
$$

\section{DSR compatibility of weakly-proper de Sitter}

Let us now comment on the DSR-relativistic compatibility of the "weakly-proper dS momentum space" which we introduced in Subsection $\mathrm{VA}$. This is the scenario we obtained adopting on momentum space de Sitter metric and its Levi-Civita connection, but then requiring that the composition law be commutative and compatible with the connection according to standard interpretation (6). Following the strategy of analysis already discussed in the previous subsection it is easy to see that also this weakly-proper dS momentum space is DSR-relativistic compatible. And the core ingredient of this relativistic compatibility is exactly the same as for the case of the proper-dS momentum space discussed in the previous subsection. Indeed both scenarios have the same on-shellness (same metric on momentum space, the de Sitter one) whose invariance is assured by (at second order in $\ell$ and at first order in the boost parameter $\xi)$

$$
\left\{\begin{array}{l}
\tilde{\Lambda}_{0}(p) \simeq p_{0}+\xi p_{1} \\
\tilde{\Lambda}_{1}(p) \simeq p_{1}+\xi\left(p_{0}-\ell p_{0}^{2}-\frac{\ell}{2} p_{1}^{2}+\frac{2}{3} \ell^{2} p_{0}^{3}\right)
\end{array}\right.
$$

The differences between the weakly-proper-dS and the proper-dS case all reside in the law of composition of momenta, which in the weakly-proper-dS case takes the form

$$
\left\{\begin{array}{l}
\left(q \oplus_{\ell} k\right)_{0}^{(2)}=q_{0}+k_{0}-\ell q_{1} k_{1} \\
\left(q \oplus_{\ell} k\right)_{1}^{(2)}=q_{1}+k_{1}-\ell\left(q_{0} k_{1}+q_{1} k_{0}\right)+\ell^{2} k_{1}\left(q_{0}^{2}+\frac{1}{2} q_{1}^{2}\right)+\ell^{2} q_{1}\left(k_{0}^{2}+\frac{1}{2} k_{1}^{2}\right)
\end{array}\right.
$$

It is noteworthy that, in spite of their differences, both this composition law for the weakly-proper-dS case and the composition law for the proper-dS are covariant under the boosts 120 . We leave to the interested reader the simple task of verifying that indeed for the weakly-proper composition law (121) one does have that

$$
\tilde{\Lambda}^{\xi}\left(q \oplus_{\ell} k\right)=\tilde{\Lambda}^{\xi}(q) \oplus_{\ell} \tilde{\Lambda}^{\xi}(k)
$$

So we have more than one (at least tw $\mathrm{g}^{3}$ commutative composition laws which can be consistently used in a DSRrelativistic picture in combination with the on-shellness obtained from the dS metric of momentum space.

\section{Aside on more general forms of DSR-relativistic compatibility}

Both for the proper-dS and for the weakly-proper-dS momentum spaces we established a form of DSR-relativistic compatibility which is still rather intuitive. This is based on requiring for the action of boosts on composed momenta that

$$
B^{\xi} \triangleright\left(q \oplus_{\ell} k\right)=\tilde{\Lambda}^{\xi}(q) \oplus_{\ell} \tilde{\Lambda}^{\xi}(k)
$$

\footnotetext{
${ }^{3}$ We here explicitly obtained two commutative composition laws (proper-dS case and weakly-proper-dS case) which satisfy this requirement of DSR-relativistic compatibility with the on-shellness obtained from the dS metric of momentum space. It goes beyond the scopes of this analysis to determine how many such commutative composition laws exist. Concerning noncommutative composition laws we here consider the single case of the $\kappa$-momentum space, with its noncommutative composition law which is also (see subsection VIE) DSR-relativistic compatible with the on-shellness obtained from the dS metric of momentum space. And as we were in the final stages of preparation of this manuscript we noticed the very recent study in Ref. [37] which provides another example of noncommutative composition law which is DSR-relativistic compatible with the on-shellness obtained from the dS metric of momentum space.
} 
with

$$
B^{\xi} \triangleright\left(q \oplus_{\ell} k\right)=\left.\tilde{\Lambda}_{\mu}^{\xi}(\mathcal{P})\right|_{\mathcal{P}=q \oplus_{\ell} k}
$$

where $p \rightarrow \tilde{\Lambda}^{\xi}(p)$ is a DSR-deformed ( $\ell$-dependent) boost map which is compatible with the momentum-space metric in the sense that it leaves the on-shellness invariant.

And this intuitive setup also allows one to describe the action of boosts on composed momenta via generators of the form

$$
\tilde{N}_{[q \oplus \ell k]}^{\xi}=\tilde{N}_{[q]}^{\xi}+\tilde{N}_{[k]}^{\xi}
$$

meaning that the action of the boost generator on the composed momenta is simply obtained by acting on each momentum in the composition with the single-momentum generator (the operator which generates the boost map $\left.p \rightarrow \tilde{\Lambda}^{\xi}(p)\right)$.

Our next task is to observe that this particularly simple structure of the DSR-relativistic properties is not representative of the most general case. One can achieve the equivalence of inertial observers ("absence of a preferred frame") also in some less intuitive and more complicated ways. We shall dwell on this not only for its intrinsic interest from a general perspective but also because the DSR-relativistic compatibility of the $\kappa$-momentum space can only be understood from within this wider picture, as we shall show in the next subsection.

We get on our way toward discussing these issues by assuming that it has been already established that some description of the boost map on the momentum of a single particle,

$$
B^{\xi} \triangleright p_{\mu}=\tilde{\Lambda}_{\mu}^{\xi}(p) \approx p_{\mu}+\xi \tilde{N}_{\mu}(p)
$$

is relativistically compatible with the on-shellness

$$
d_{\ell}^{2}\left(B^{\xi} \triangleright p\right)=d_{\ell}^{2}(p)=m_{p}^{2}
$$

The part which can be highly non-trivial [4, 18, 24, 25] is the formulation of DSR-relativistic properties for the law of composition of momenta, i.e. concerns the action of boosts on composed momenta: $B_{\xi} \triangleright\left(q \oplus_{\ell} k\right)$. It should be observed that one obtains a DSR-relativistic picture if the following properties are established:

- A first requirement is that the law of composition of momenta be "covariant in substance", by which we mean that the action of boosts on composed momenta should be such that conservation laws are observer independent. For the case of a two-body particle decay this takes the shape of the requirement

$$
p=q \oplus_{\ell} k \Rightarrow B^{\xi} \triangleright p=B^{\xi} \triangleright\left(q \oplus_{\ell} k\right)
$$

Evidently this requirement is satisfied if the action of boosts on a momentum obtained combining two other momenta follows the same law of transformation of a corresponding single momentum:

$$
B^{\xi} \triangleright\left(q \oplus_{\ell} k\right)=\left.\tilde{\Lambda}_{\mu}^{\xi}(\mathcal{P})\right|_{\mathcal{P}=q \oplus_{\ell} k}
$$

- A second requirement is that the law of composition of momenta be "covariant in form", by which we mean that the action of boosts on momenta composed via the $\oplus_{\ell}$ rule produces momenta which are still composed via the $\oplus_{\ell}$ rule. We make explicit this requirement by introducing notation $p^{[A]}, q^{[A]}$ for the momenta of two particles measured by Alice as part of a composition law (momenta of particles taking part in an event, and therefore entering a law of conservation of energy-momentum) and notation $p^{[B]}, q^{[B]}$ for the momenta of those same two particles as determined by observer Bob, purely boosted with respect to Alice. The requirement then takes the form of

$$
B^{\xi} \triangleright\left(q^{[A]} \oplus_{\ell} k^{[A]}\right)=q^{[B]} \oplus_{\ell} k^{[B]}
$$

- As third requirement we shall insist on having that $q^{[B]}$ (respectively $k^{[B]}$ ) is on the same shell as $q^{[A]}($ respectively $\left.k^{[A]}\right)$.

The first requirement ensures that processes which are allowed for observer Alice (i.e. such that momentum is indeed conserved for Alice) are also allowed for Bob (i.e. momentum is conserved also according to Bob). Moreover, Alice and Bob agree (because of the second requirement) on the form of the composition law and also agree on which particles take part in the process (third requirement). So there is no way to distinguish between Alice and Bob: the laws of kinematics are exactly the same for Alice and Bob, as required for a relativistic picture. 
The intuitive case of DSR-relativistic pictures which we encountered for the proper-dS and weakly-proper-dS cases satisfies these requirements in a particularly simple way: the boost $B^{\xi}$ of rapidity $\xi$ acts on composed momenta in such a way that $q^{[B]}$ is just $\tilde{\Lambda}_{\mu}^{\xi}\left(q^{[A]}\right)$ and $k^{[B]}$ is just $\tilde{\Lambda}_{\mu}^{\xi}\left(k^{[A]}\right)$. This evidently ensures that, as dictated by the third requirement, $q^{[B]}$ (respectively $k^{[B]}$ ) is on the same shell as $q^{[A]}$ (respectively $k^{[A]}$ ). But there are more general ways to ensure the third requirement, while not spoiling the first and second requirement. In particular, one can allow for $q^{[B]}$ to be $\tilde{\Lambda}_{\mu}^{\xi_{1}}\left(q^{[A]}\right)$ and $k^{[B]}$ to be $\tilde{\Lambda}_{\mu}^{\xi_{2}}\left(k^{[A]}\right)$, with any choice of $\xi_{1}$ and $\xi_{2}$, and still the third requirement is satisfied:

$$
B^{\xi} \triangleright\left(q^{[A]} \oplus_{\ell} k^{[A]}\right)=\tilde{\Lambda}^{\xi_{1}}\left(q^{[A]}\right) \oplus_{\ell} \tilde{\Lambda}^{\xi_{2}}\left(k^{[A]}\right)
$$

The $\kappa$-momentum space will give us an explicit example where this is the way in which the third requirement is satisfied, preserving the compatibility with the first and second requirement. As we shall see is the case of $\kappa$-momentum space, one in general expects $\xi_{1}$ and $\xi_{2}$ to be proportional to the rapidity parameter $\xi$ of the boost, but having $\xi_{1}=\xi=\xi_{2}$ is not the only admissible way (for the $\kappa$-momentum space we shall find that $\xi_{1}=\xi$ but $\xi_{2} \neq \xi$ ). Of course any difference between $\xi_{1}$ and $\xi$ and/or between $\xi_{2}$ and $\xi$ must be governed by the only relevant physical observables, which are the momenta which are being composed.

In closing this aside we also notice that 129 can be rewritten for generators in the following way

$$
\tilde{N}_{[q \oplus \ell k]}^{\xi}=\tilde{N}_{[q]}^{\xi_{1}}+\tilde{N}_{[k]}^{\xi_{2}}
$$

a special case of which is 123 .

\section{E. DSR-relativistic compatibility of $\kappa$-dS momentum space}

We are now ready for the task of establishing the DSR-relativistic compatibility of the $\kappa$-dS momentum space. We first note that the action of a Lorentz boost on single momenta $\tilde{\Lambda}_{\mu}^{\xi}(p)$ is the same we encountered for the "proper-dS" and "weakly-proper-dS" momentum spaces:

$$
\left\{\begin{array}{l}
\tilde{N}_{0}(p)=p_{1} \\
\tilde{N}_{1}\left(p_{0}\right)=\left(\frac{1}{2 \ell}\left(1-e^{-2 \ell p_{0}}\right)-\frac{\ell}{2} p_{1}^{2}\right)
\end{array}\right.
$$

This is due to the fact that also on the $\kappa$-dS momentum space the metric is the de Sitter metric (so the action of boosts on single momenta must enforce the observer independence of the same on-shellness). The differences are all at the level of the composition law, and particularly significant for our purposes is the fact that on the $\kappa$-dS momentum space the composition law is noncommutative:

$$
\left\{\begin{array}{l}
\left(q \oplus_{\ell} k\right)_{0}=q_{0}+k_{0} \\
\left(q \oplus_{\ell} k\right)_{1}=q_{1}+k_{1} e^{-\ell q_{0}}
\end{array}\right.
$$

This noncommutativity is evidently a challenge [18] for a symmetric rule of action of boosts on composed momenta, of the type $B^{\xi} \triangleright\left(q \oplus_{\ell} k\right)=\tilde{\Lambda}^{\xi}(q) \oplus_{\ell} \tilde{\Lambda}^{\xi}(k)$. We shall see that the $\kappa$-dS momentum space is DSR-relativistic compatible but only according to the sort of relativistic prescriptions described in the previous subsection, such that

$$
B^{\xi} \triangleright\left(q \oplus_{\ell} k\right) \neq \tilde{\Lambda}^{\xi}(q) \oplus_{\ell} \tilde{\Lambda}^{\xi}(k)
$$

Building on results of previous studies of the $\kappa$-dS momentum space [18, 27, 28, we shall give a more detailed account than ever before of the relativistic properties of the $\kappa$-dS momentum space, when adopting as rule of action on the composition of two momenta the following [28]

$$
B^{\xi} \triangleright\left(q \oplus_{\ell} k\right)=\tilde{\Lambda}^{\xi}(q) \oplus_{\ell} \tilde{\Lambda}^{\xi e^{-\ell q_{0}}}(k)
$$

where we are here satisfied with working at leading order 4 in $\xi$ and the symbol $\tilde{\Lambda}^{\xi e^{-\ell q_{0}}}$ is intended as just one of our deformed boosts but with the peculiarity that the rapidity parameter is specified not only in terms of the rapidity

\footnotetext{
${ }^{4}$ A generalization of 134 to all orders in $\xi$ can be found in Ref. [28.
} 
parameter connecting the two relevant observers but also in terms of the (exponential of the zeroth component of) the momentum appearing on the left-hand side of the composition law.

It is important to notice that (134) automatically enforces the "covariance in form" characterized, as stressed earlier, by the requirement $B^{\xi} \triangleright\left(q^{[A]} \oplus_{\ell} k^{[A]}\right)=q^{[B]} \oplus_{\ell} k^{[B]}$ : for this one should interpret (134) as establishing that the momenta determined by the two observers, Alice and Bob, connected by the boosts are related according to $q^{[B]}=\tilde{\Lambda}^{\xi}\left(q^{[A]}\right)$ and $k^{[B]}=\tilde{\Lambda}^{\xi e^{-\ell q_{0}}}\left(k^{[A]}\right)$. Notice that adopting this interpretation one still has (since the only actions are via $\tilde{\Lambda}$, which by construction leaves the shell unchanged) that $k^{[B]}$ and $k^{[A]}$ (and respectively $q^{[B]}$ and $q^{[A]}$ ) are on the same mass shell, as necessary for relativistic invariance of the theory. Our remaining task is to verify that our formulation of relativistic transformations of $\kappa$ - $\mathrm{dS}$ composed momenta is "covariant in substance", in the sense specified earlier through the requirement $B^{\xi} \triangleright\left(a \oplus_{\ell} b\right)=\left.\tilde{\Lambda}_{\mu}^{\xi}(\mathcal{P})\right|_{\mathcal{P}=a \oplus_{\ell} b}$ or equivalently in the $\kappa$-dS case (also taking in to account (134)) the requirement [18, 27.

$$
\left.\xi \tilde{N}_{\mu}(\mathcal{P})\right|_{\mathcal{P}=q \oplus_{\ell} k}=\xi \frac{\partial}{\partial q_{\rho}}\left(q \oplus_{\ell} k\right)_{\mu} \tilde{N}_{\rho}(q)+\xi e^{-\ell q_{0}} \frac{\partial}{\partial k_{\rho}}\left(q \oplus_{\ell} k\right)_{\lambda} \tilde{N}_{\rho}(k)
$$

Before verifying this 135 let us pause briefly for observing that Eq. (134) and Eq. (135) provide intuition for the nature of the peculiarities of the $\kappa$-dS momentum space: Eq. (134) lends itself to the perspective of how boosts act on each of the momenta in the composition law and shows that for the second of the two momenta the boost parameter is affected by a "back reaction" 28] such that the first momentum in the composition law enters in the rapidity parameter "felt by" the second momentum in the composition law; instead Eq. 135 lends itself to the perspective of how the action of boost generators on composed momenta is structured in terms of the action of boosts generators on single momenta (in this case a rule of action of the type $\tilde{N}_{\left[q \oplus_{\ell} k\right]}^{\xi}=\tilde{N}_{[q]}^{\xi}+e^{-\ell q_{0}} \tilde{N}_{[k]}^{\xi}$ ) that leads to the notion [18, 27] of a "law of composition of boosts".

Turning back to the "covariance in substance" of this $\kappa$-dS case, we opt for structuring our proof in such a way that we can expose the uniqueness of the option 135 . We do so by considering at first the slightly more general requirement

$$
\left.\xi \tilde{N}_{\mu}(\mathcal{P})\right|_{\mathcal{P}=q \oplus_{\ell} k}=\xi_{(1)} \frac{\partial}{\partial q_{\rho}}\left(q \oplus_{\ell} k\right)_{\mu} \tilde{N}_{\rho}(q)+\xi_{(2)} \frac{\partial}{\partial k_{\rho}}\left(q \oplus_{\ell} k\right)_{\lambda} \tilde{N}_{\rho}(k)
$$

so that we can find as a result of the analysis that this requirement can only be satisfied with the choice of $\xi_{(1)}$ and $\xi_{(2)}$ made in 135 , i.e. $\xi_{(1)}=\xi$ and $\xi_{(2)}=e^{-\ell q_{0}} \xi$.

For the zeroth component of 136 , using the form of our $\tilde{N}$ one easily finds that:

$$
\left\{\begin{array}{l}
\left.\xi \tilde{N}_{0}(\mathcal{P})\right|_{\mathcal{P}=q \oplus_{\ell} k}=\xi\left(q \oplus_{\ell} k\right)_{1}=\xi\left(q_{1}+k_{1} e^{-\ell q_{0}}\right) \\
\xi_{(1)} \frac{\partial}{\partial q_{\rho}}\left(q \oplus_{\ell} k\right)_{0} \tilde{N}_{\rho}(q)+\xi_{(2)}(\xi, q) \frac{\partial}{\partial k_{\rho}}\left(q \oplus_{\ell} k\right)_{0} \tilde{N}_{\rho}(k)=\xi_{(1)} q_{1}+\xi_{(2)} k_{1}
\end{array}\right.
$$

From this one sees that the requirement

$$
\left(\xi-\xi_{(1)}\right) q_{1}+\left(\xi e^{-\ell q_{0}}-\xi_{(2)}\right) k_{1}=0
$$

must be satisfied, which in turn leads us to the sought conclusion $\xi_{(1)}=\xi, \xi_{(2)}=\xi e^{-\ell q_{0}}$ as the only option for having 136) verified for arbitrary $q$ and $k$.

Let us then reinstate $\xi_{(1)}=\xi, \xi_{(2)}=\xi e^{-\ell q_{0}}$ in 136 and verify that also the 1 component of 136 is satisfied. For this we observe that on the left-hand side of 136 we have

$$
\begin{gathered}
\left.\tilde{N}_{1}(\mathcal{P})\right|_{\mathcal{P}=q \oplus_{\ell} k}=\left.\left(\frac{1}{2 \ell}\left(1-e^{-2 l \ell P_{0}}\right)-\frac{\ell}{2} \mathcal{P}_{1}^{2}\right)\right|_{\mathcal{P}=q \oplus_{\ell} k}=\frac{1}{2 \ell}\left(1-e^{-2 \ell\left(q_{0}+k_{0}\right)}\right)-\frac{\ell}{2}\left(q_{1}+k_{1} e^{-\ell q_{0}}\right)^{2}= \\
=\frac{1}{2 \ell}\left(1-e^{-2 \ell\left(q_{0}+k_{0}\right)}\right)-\frac{\ell}{2}\left(q_{1}+k_{1} e^{-\ell q_{0}}\right)^{2}
\end{gathered}
$$

while for the right-hand side we obtain

$$
\begin{gathered}
\frac{\partial}{\partial q_{\theta}}\left(q \oplus_{\ell} k\right)_{1} \tilde{N}_{\theta}(q)+e^{-\ell q_{0}} \frac{\partial}{\partial k_{\theta}}\left(q \oplus_{\ell} k\right)_{1} \tilde{N}_{\theta}(k)= \\
=\frac{\partial}{\partial q_{\theta}}\left(q_{1}+k_{1} e^{-\ell q_{0}}\right) \tilde{N}_{\theta}(q)+e^{-\ell q_{0}} \frac{\partial}{\partial k_{\theta}}\left(q_{1}+k_{1} e^{-\ell q_{0}}\right) \tilde{N}_{\theta}(k)= \\
=\left(\tilde{N}_{1}(q)-\ell k_{1} e^{-\ell q_{0}} \tilde{N}_{0}(q)\right)+e^{-2 \ell q_{0}} \tilde{N}_{1}(k)= \\
=\frac{1}{2 \ell}\left(1-e^{-2 \ell\left(q_{0}+k_{0}\right)}\right)-\frac{\ell}{2}\left(q_{1}+k_{1} e^{-\ell q_{0}}\right)^{2}
\end{gathered}
$$


So, as desired, the left-hand side of (136), which is (139), agrees with the right-hand side of (136), which is (140).

This concludes our investigation of the relativistic properties of the $\kappa$-dS momentum space. Evidently these relativistic properties are technically sound but it does appear that the condition $k^{[B]}=\tilde{\Lambda}^{\xi e^{-\ell q_{0}}}\left(k^{[A]}\right)$ may require an additional effort of interpretation. That condition essentially implies that the same kind of particle with the same value of momentum $k$ should transform differently depending on whether the particle is freely propagating (no role in any conservation law) or it enters in an interaction event (so that its momentum $k$ gets composed to other momenta), with in turn different interaction events producing different transformation laws for $k$. Dwelling on whether or not such an interpretation leads to "acceptable" physical predictions goes beyond the scopes of our analysis: the study of $\kappa$-dS momentum space is a lively research area and we felt it could be a valuable contribution to its development if we clearly exposed the strengths and the peculiarities of its relativistic properties. Our findings for the DSR-relativistic properties of the $\kappa$-dS momentum space provide a solid technical basis on which future investigations of the suitability for physics of this momentum space may rely.

While postponing to future studies a more through analysis of the "physical acceptability" of the relativistic properties of the $\kappa$-dS momentum space, we close this section highlighting a few facts that confirm that at least technically we do have here a fully relativistic framework. For this purpose let us start by observing that the results reported above show that two observers, Alice and Bob, connected by a pure boost, witnessing energy-momentum conservation in a particle-physics process, agree on the mass shell to which each particle belongs and also agree on the form of the law of energy-momentum conservation (they agree on the form of $\oplus_{\ell}$ ). Moreover, one should notice that the $\kappa$-dS rules of transformation between Alice and Bob are truly relational (no preferred observer), as one easily sees by considering how the map from Bob to Alice is related to the map from Alice to Bob. For this take Alice with momenta $q^{[A]}$ and $k^{[A]}$ entering an event through a composition $q^{[A]} \oplus_{\ell} k^{[A]}$. And take the map from Alice to Bob to be characterized by rapidity/boost parameter $\xi$, so that Bob has $q^{[B]}=\tilde{\Lambda}^{\xi}\left(q^{[A]}\right)$ and $k^{[B]}=\tilde{\Lambda}^{\xi e^{-\ell q_{0}^{[A]}}}\left(k^{[A]}\right)$. If we now look at the inverse situation, starting with Bob's $q^{[B]}$ and $k^{[B]}$ entering an event through a composition $q^{[B]} \oplus_{\ell} k^{[B]}$, then the map from Bob to Alice is characterized by boost parameter $-\xi$, and Alice finds $q^{[A]}=\tilde{\Lambda}^{-\xi}\left(q^{[B]}\right)$ and $k^{[A]}=\tilde{\Lambda}^{-\xi e^{-\ell q_{0}^{[B]}}}\left(k^{[B]}\right)$. Evidently this does give a consistent picture as verified explicitly to leading order in $\xi$ through the following observation

$$
\begin{aligned}
& \tilde{\Lambda}^{\xi e^{-\ell q_{0}^{[A]}}}\left(\tilde{\Lambda}^{-\xi e^{-\ell q_{0}^{[B]}}}\left(k^{[B]}\right)\right) \approx \\
& \approx \tilde{\Lambda}^{-\xi e^{-\ell q_{0}^{[B]}}}\left(k^{[B]}\right)+\xi e^{-\ell q_{0}^{[A]}} N^{(\ell)}\left(\tilde{\Lambda}^{-\xi e^{-\ell q_{0}^{[B]}}}\left(k^{[B]}\right)\right) \approx \\
& \approx k^{[B]}-\xi e^{-\ell q_{0}^{[B]}} \tilde{N}\left(k^{[B]}\right)+\xi e^{-\ell q_{0}^{[A]}} \tilde{N}\left(\tilde{\Lambda}^{-\xi e^{-\ell q_{0}^{[B]}}}\left(k^{[B]}\right)\right) \approx \\
& \approx k^{[B]}-\xi e^{-\ell q_{0}^{[B]}} \tilde{N}\left(k^{[B]}\right)+\xi e^{-\ell q_{0}^{[A]}} \tilde{N}\left(k^{[B]}\right)=k^{[B]}
\end{aligned}
$$

where we also used the fact that $\xi\left(q^{[B]}-q^{[A]}\right)=0+O\left(\xi^{2}\right)$.

\section{RELATIVISTIC CONSISTENCY AS A GEOMETRIC PROPERTY}

In the previous sections we reported some new results that can have direct applicability in future studies of the $\kappa$-dS, the proper-dS and the weakly-proper-dS momentum spaces. In this section we want to offer an additional contribution to the conceptual characterization of some of our results, by considering momentum-space diffeomorphisms.

A significant part of our analysis was focused on the requirements necessary for (DSR-)relativistic covariance of theories on momentum space. It remains unclear whether specifically the formulation of such theories within the relative-locality framework of Ref. [9] should also be invariant under momentum-space diffeomorphisms, in the sense of general covariance: this appears at first sight desirable but several grey areas of understanding remain concerning what happens to these theories when we change coordinates on momentum space. Our main objective in this section is to provide evidence of the fact that, while other properties of momentum-space theories may well depend on the choice of momentum-space coordinatization, the property of a momentum space of being DSR-relativistic compatible is a truly geometric property. We do this by considering our proper-dS momentum space, with the properties established within a chosen coordinatization of momentum spac $~_{5}^{5}$ in the previous sections and showing that under the action of a momentum-space diffeomorphism one obtains a different coordinatization of the proper-dS momentum space which still satisfies the requirements of DSR-relativistic compatibility.

\footnotetext{
${ }^{5}$ We note in passing that the coordinatization of proper-dS momentum space adopted in the previous sections reproduces in leading order in $\ell$ the results for a DSR-relativistic setup of kinematics reported in Ref. 4]. In retrospect one can therefore view the results of Ref. 4] as results at leading order in $\ell$ for the proper-dS momentum space.
} 
The results in this section also contribute to the interesting issue, already explored in Refs. [18, 19], that concerns whether the property of theories of being (DSR-)relativistic can be fully encoded as a momentum-space property.

At the end of this section we also use tools that become available when considering momentum-space diffeomophisms to establish the DSR-relativistic compatibility of proper-dS momentum space to all orders in $\ell$ (in the previous sections this was only established at order $\ell^{2}$ ).

Because of the nature of the manipulations performed in this section we find convenient to drop the label $\ell$, leaving the $\ell$-dependence of quantities implicit. Interested readers can restore $\ell$ by simple use of dimensional analysis.

And, again because of the nature of the manipulations performed in this section, we find convenient to use the notation

$$
\left.\tilde{\Lambda}\left(q \oplus_{\ell} k\right) \equiv \tilde{\Lambda}(\mathcal{P})\right|_{\mathcal{P}=q \oplus_{\ell} k}
$$

The DSR-deformed boost transformations $\tilde{\Lambda}$ are functions defined on single momenta, and we are extending their definition to a momentum obtained by composing two other momenta.

\section{A. Diffeomorphisms of proper-dS momentum space}

Let us then consider our proper-dS momentum space, with its symmetry transformation $S$, which contain in particular the subset of DSR-deformed boost transformations $\tilde{\Lambda}$ :

$$
\left\{\begin{array}{l}
d^{2}(S(k), S(q))=d^{2}(k, q) \\
\tilde{\Lambda}\left(q \oplus_{\Gamma} k\right)=\tilde{\Lambda}(q) \oplus_{\Gamma} \tilde{\Lambda}(k)
\end{array}\right.
$$

where we changed notation from $\oplus_{\ell}$ to $\oplus_{\Gamma}$ just as a way to stress the role of the connection $\Gamma$ in the law of composition of momenta. This role of the connection will be at center stage in this section.

Next we consider a diffeomorphism:

$$
\left\{\begin{array}{l}
p_{\lambda} \rightarrow p_{\lambda}^{\prime}=f_{\lambda}(p) \\
V_{\lambda}(p) \rightarrow V_{\lambda}^{\prime}\left(p^{\prime}\right)=J_{\lambda}^{\alpha} V_{\alpha}(p) \\
g^{\mu \nu}(p) \rightarrow g^{\prime \mu \nu}\left(p^{\prime}\right)=\bar{J}_{\alpha}^{\mu} \bar{J}_{\beta}^{\nu} g^{\alpha \beta}(p) \\
\Gamma_{\lambda}^{\mu \nu}(p) \rightarrow \Gamma^{\prime \mu \nu}{ }_{\lambda}\left(p^{\prime}\right)=\bar{J}_{\gamma}^{\nu} \bar{J}_{\rho}^{\mu} J_{\lambda}^{\beta} \Gamma_{\beta}^{\rho \gamma}(p)-\bar{J}_{\gamma}^{\nu} \bar{J}_{\rho}^{\mu}\left(\partial^{\rho} J_{\lambda}^{\gamma}\right)
\end{array}\right.
$$

where $V_{\lambda}(p)$ is a generic vector field, $J(p)$ is the Jacobi matrix $J_{\lambda}^{\alpha}(p)=\partial^{\alpha} f_{\lambda}(p)$ and $\bar{J}$ is the inverse of $J$.

We are interested in establishing that in the new coordinates one still has a set of symmetries $S^{\prime}$ and a subset $\tilde{\Lambda}^{\prime}$ such that

$$
\left\{\begin{array}{l}
d^{\prime 2}\left(S^{\prime}\left(k^{\prime}\right), S^{\prime}\left(q^{\prime}\right)\right)=d^{2}\left(k^{\prime}, q^{\prime}\right) \\
\tilde{\Lambda}^{\prime}\left(q^{\prime} \oplus_{f(\Gamma)}^{[f(0)]} k^{\prime}\right)=\tilde{\Lambda}^{\prime}\left(q^{\prime}\right) \oplus_{f(\Gamma)}^{[f(0)]} \tilde{\Lambda}^{\prime}\left(k^{\prime}\right)
\end{array}\right.
$$

where $d^{\prime 2}$ is the distance function associated to the transformed metric $g^{\prime \mu \nu}$ and $\oplus_{f(\Gamma)}^{[f(0)]}$ is the translated composition law that we defined in $(18)$, associated to the subtraction point $f(0)$ and to the transformed connection:

$$
f(\Gamma)_{\lambda}^{\mu \nu}=\Gamma_{\lambda}^{\prime \mu \nu}\left(p^{\prime}\right)=\bar{J}_{\gamma}^{\nu} \bar{J}_{\rho}^{\mu} J_{\lambda}^{\beta} \Gamma_{\beta}^{\rho \gamma}(p)-\bar{J}_{\gamma}^{\nu} \bar{J}_{\rho}^{\mu}\left(\partial^{\rho} J_{\lambda}^{\gamma}\right)
$$

For what concerns the invariance of the distance function, we can exploit again the fact that it is equivalent to considering the invariance of the line element:

$$
g^{\mu \nu}(S(p)) d S_{\mu}(p) d S_{\nu}(p)=g^{\mu \nu}(p) d p_{\mu} d p_{\nu}
$$

We then must seek the transformations $S^{\prime}$ such that

$$
g^{\prime \mu \nu}\left(S^{\prime}\left(p^{\prime}\right)\right) d S_{\mu}^{\prime}\left(p^{\prime}\right) d S_{\nu}^{\prime}\left(p^{\prime}\right)=g^{\prime \mu \nu}\left(p^{\prime}\right) d p_{\mu}^{\prime} d p_{\nu}^{\prime}
$$

Using the transformation properties of the metric, of $S$ and of the differential of a vector we can notice that

$$
g^{\prime \mu \nu}\left(S^{\prime}\left(p^{\prime}\right)\right) d S_{\mu}^{\prime}\left(p^{\prime}\right) d S_{\nu}^{\prime}\left(p^{\prime}\right)=g^{\alpha \beta}(S(p)) d S_{\mu}(p) d S_{\nu}(p)
$$


Then, with 146 and using again the transformation properties of the metric we get

$$
g^{\alpha \beta}(S(p)) d S_{\mu}(p) d S_{\nu}(p)=g^{\mu \nu}(p) d p_{\mu} d p_{\nu}=g^{\prime \mu \nu}\left(p^{\prime}\right) d p_{\mu}^{\prime} d p_{\nu}^{\prime}
$$

which is the result we were seeking.

Our next and final task for establishing that the DSR-relativistic compatibility of proper-dS momentum space is a diffeomorphism-invariant property concerns the law of composition of momenta. For this purpose we start from establishing that under a diffeomorphism the composition law transforms as follows:

$$
f\left(q \oplus_{\Gamma} k\right)=f(q) \oplus_{f(\Gamma)}^{[f(0)]} f(k)
$$

We can easily verify this property adopting the formulation of the role of the affine connection in the composition law which we introduced in Section II

$$
\left\{\begin{array}{l}
\frac{d \zeta_{\rho}}{d t} \nabla_{\Gamma}^{\rho} \frac{d \gamma_{\lambda}}{d s}=0 \\
q \oplus_{\Gamma} k=\gamma(1,1)
\end{array}\right.
$$

where it should be appreciated that $\gamma$ is completely determined by the affine connection $\Gamma$, the points $q$ and $k$ on momentum space and implicitly by the choice of the origin as subtraction point. In particular we have that

$$
\left\{\begin{array}{l}
\gamma(0,1)=q \\
\gamma(1,0)=k \\
\gamma(0,0)=0
\end{array}\right.
$$

A diffeomorphism $p \rightarrow f(p)$ maps the surface $\gamma$ into a surface $\gamma^{\prime}$ characterized by

$$
\gamma^{\prime}(s, t)=f(\gamma(s, t))
$$

for which in particular on has that

$$
\left\{\begin{array}{l}
\gamma^{\prime}(0,1)=f(\gamma(0,1))=f(q) \\
\gamma^{\prime}(1,0)=f(\gamma(1,0))=f(k) \\
\gamma^{\prime}(1,1)=f(\gamma(1,1))=f\left(q \oplus_{\Gamma} k\right) \\
\gamma^{\prime}(0,0)=f(0)
\end{array}\right.
$$

One can observe that, if we change the connection $\Gamma$ and the vectors $\frac{d \zeta_{\rho}}{d t}$ and $\frac{d \gamma \rho}{d t}$ according to 143 , from 150 it follows that

$$
\frac{d \zeta_{\rho}^{\prime}}{d t} \nabla_{\Gamma^{\prime}}^{\rho} \frac{d \gamma_{\lambda}^{\prime}}{d s}=0
$$

where the apices refers to the transformed quantities.

Therefore we have that $\gamma^{\prime}$ is the surface which solves 154 with the transformed connection $\Gamma^{\prime}$ and whose boundary conditions are determined by the points $q^{\prime}=f(q), k^{\prime}=f(k)$ and $\mathrm{f}(0)$ as the subtraction point. So, using the definition (18) of the translated composition law we get that:

$$
\gamma^{\prime}(1,1)=f(q) \oplus_{f(\Gamma)}^{[f(0)]} f(k)
$$

which indeed confirms $f\left(q \oplus_{\Gamma} k\right)=f(q) \oplus_{f(\Gamma)}^{[f(0)]} f(k)$, as announced in 149 .

This result provides the key ingredient for us to show that, as announced

$$
\tilde{\Lambda}^{\prime}\left(q^{\prime} \oplus_{f(\Gamma)}^{[f(0)]} k^{\prime}\right)=\tilde{\Lambda}^{\prime}\left(q^{\prime}\right) \oplus_{f(\Gamma)}^{[f(0)]} \tilde{\Lambda}^{\prime}\left(k^{\prime}\right)
$$

For this purpose we observe that

$$
\tilde{\Lambda}^{\prime}\left(p^{\prime}\right)=f(\tilde{\Lambda}(p))
$$

from which, using (149) and (157), we obtain

$$
\tilde{\Lambda}^{\prime}\left(q^{\prime} \oplus_{f(\Gamma)}^{[f(0)]} k^{\prime}\right)=\tilde{\Lambda}^{\prime}\left(f\left(q \oplus_{\Gamma} k\right)\right)=f\left(\tilde{\Lambda}\left(q \oplus_{\Gamma} k\right)\right)
$$


We then use the covariance of the composition law that we assume to hold for the untransformed coordinates

$$
f\left(\tilde{\Lambda}\left(q \oplus_{\Gamma} k\right)\right)=f\left(\tilde{\Lambda}(q) \oplus_{\Gamma} \tilde{\Lambda}(k)\right)
$$

again in combination with 149 and 157 , to establish that

$$
f\left(\tilde{\Lambda}(q) \oplus_{\Gamma} \tilde{\Lambda}(k)\right)=f(\tilde{\Lambda}(q)) \oplus_{f(\Gamma)}^{[f(0)]} f(\tilde{\Lambda}(k))=\tilde{\Lambda}^{\prime}\left(q^{\prime}\right) \oplus_{f(\Gamma)}^{[f(0)]} \tilde{\Lambda}^{\prime}\left(k^{\prime}\right)
$$

The last three equations confirm the validity of 156 , and this completes our proof of the fact that the DSR-relativistic compatibility of proper-dS momentum space is a diffeomorphism-invariant property.

\section{B. All-order DSR-relativistic compatibility of proper-dS momentum space}

Some of these techniques based on diffeomorphism transformations also allow us to establish, as announced, the DSR-relativistic compatibility of proper-dS momentum space to all orders in $\ell$. In this section we are keeping the $\ell$ dependence implicit, but the validity of results to all order in $\ell$ will of course be evident from the fact that we establish exact results.

Let us start building this proof by first using some results of the previous subsection for introducing some requirements that ensure the DSR-relativistic compatibility of the composition law. The first requirement is that the origin of momentum space is invariant under boosts $(f(0)=0)$, as in the proper-dS case. Using (149) this ensures that

$$
\tilde{\Lambda}\left(q \oplus_{\Gamma} k\right)=\tilde{\Lambda}(q) \oplus_{\Gamma^{\prime}} \tilde{\Lambda}(k)
$$

where $\Gamma^{\prime}$ is

$$
\Gamma_{\alpha}^{\prime \mu \rho}\left(p^{\prime}\right) \equiv \bar{J}_{\gamma}^{\rho} \bar{J}_{\nu}^{\mu} J_{\alpha}^{\beta} \Gamma_{\beta}^{\nu \gamma}(p)-\bar{J}_{\gamma}^{\rho} \bar{J}_{\nu}^{\mu}\left(\partial^{\nu} J_{\alpha}^{\gamma}\right)
$$

$J$ is the Jacobian $J_{\alpha}^{\mu}=\partial^{\mu} \tilde{\Lambda}_{\alpha}$ and $\bar{J}$ is the inverse of $J$. The other requirement, also evidently satisfied in the proper-dS case, is the invariance of the connection under boosts $\left(\Gamma^{\prime}=\Gamma\right)$. When both requirements are satisfied the covariance of the composition law is indeed guaranteed, since combining invariance of the connection with 161 one has that

$$
\Gamma^{\prime}=\Gamma \rightarrow \tilde{\Lambda}\left(q \oplus_{\Gamma} k\right)=\tilde{\Lambda}(q) \oplus_{\Gamma} \tilde{\Lambda}(k)
$$

Having established this powerful point about the DSR-relativistic compatibility of composition laws, let us now consider the case of a suitable momentum space metric $g$, such that a DSR-deformed boost $\tilde{\Lambda}$ is a diffeomorphism on momentum space with the property

$$
d^{2}(\tilde{\Lambda}(p), \tilde{\Lambda}(q))=d^{2}(p, q)
$$

which is equivalent to the condition of invariance of the metric:

$$
g^{\mu \nu}\left(p^{\prime}\right)=g^{\prime \mu \nu}\left(p^{\prime}\right)=\bar{J}_{\alpha}^{\mu} \bar{J}_{\beta}^{\nu} g^{\alpha \beta}(p)
$$

If then, as in the proper-dS case, one adopts as composition law the one produced, according to our novel geometric interpretation, by the Levi-Civita connection of the metric $g$

$$
A_{\alpha}^{\mu \rho}(p)=\frac{1}{2} \bar{g}_{\alpha \beta}(p)\left(g^{\beta \mu, \rho}(p)+g^{\beta \rho, \mu}(p)-g^{\mu \rho, \beta}(p)\right)
$$

the overall DSR-relativistic compatibility is guaranteed. In fact, it is easy to see that the Levi-Civita connection is always invariant under the deformed boosts $\tilde{\Lambda}$,

$$
A_{\alpha}^{\prime \mu \rho}\left(p^{\prime}\right)=\frac{1}{2} \bar{g}_{\alpha \beta}^{\prime}\left(p^{\prime}\right)\left(g^{\prime \beta \mu, \rho}\left(p^{\prime}\right)+g^{\prime \beta \rho, \mu}\left(p^{\prime}\right)-g^{\prime \mu \rho, \beta}\left(p^{\prime}\right)\right)=\frac{1}{2} \bar{g}_{\alpha \beta}\left(p^{\prime}\right)\left(g^{\beta \mu, \rho}\left(p^{\prime}\right)+g^{\beta \rho, \mu}\left(p^{\prime}\right)-g^{\mu \rho, \beta}\left(p^{\prime}\right)\right)=A^{\mu \rho}\left(p^{\prime}\right),
$$

which in turn, using (163), leads us to our sought result:

$$
\tilde{\Lambda}(q) \oplus_{A^{\prime}} \tilde{\Lambda}(k)=\tilde{\Lambda}(q) \oplus_{A} \tilde{\Lambda}(k)
$$


This concludes our proof of exact (DSR-)relativistic invariance. And it should be noticed that in this subsection we did not make use of any result based on the specific form of the dS metric and/or its Levi-Civita connection. So evidently our proof of (DSR-)relativistic invariance applies to a wider class of momentum spaces: when adopting the novel interpretation of the composition law which we here introduced in 13 - 15 one gets a DSR-relativisticcompatible setup whenever the metric is DSR-compatible (in the sense of (164)) and the composition law is formulated using (in the sense of the novel geometric interpretation we here introduced through Eqs. (13)-(15)) its associated LeviCivita connection. Certain other choices of momentum-space affine connection are also DSR-relativistic compatible with such a momentum-space metric (see, e.g., the case of $\kappa$-dS momentum space), but the specific possibility of pairing the metric with its Levi-Civita connection ensures the overall DSR-relativistic compatibility. We feel that this should be viewed as an aspect of compellingness of our geometric interpretation centered on (13)- 15 .

\section{OUTLOOK}

In closing we offer a few remarks on what we see as the most significant potential implications of the results we here reported.

The most direct implications are for the study of the recently-proposed relative-locality framework. The momentum space which has been so far most studied within this framework is the $\kappa$-dS momentum space. And we here provided several additional tools for the investigation of this possibility, particularly through our analysis of relativistic properties, which went in greater depth than any such previous analysis of the $\kappa$-dS momentum space.

The appeal of the $\kappa$-dS momentum space resides (among other things) in the associativity of the composition law. But the affine connections that provide associative composition laws are a small subset of those one could in principle consider for the relative-locality framework, so in that respect the $\kappa$-dS momentum space is not representative of the possible structure of theories on curved momentum spaces. Moreover, the $\kappa$-dS momentum space has a noncommutative law of composition of momenta, which is not a general property of curved momentum spaces and is also not necessarily a "desirable" property (the associated interpretational challenges can be handled [9], but indeed they are not necessarily desirable).

The proper-dS and the weakly-proper-dS momentum spaces which we here introduced are usefully complementary to the $\kappa$-dS momentum space, at least in the sense that they have non-associative but commutative laws of composition of momenta. We were here primarily focusing on the structure and relativistic properties of these momentum spaces. Future works should establish what are the observable differences between cases with associative but noncommutative composition laws and cases with non-associative but commutative composition laws.

These new cases with non-associative but commutative composition laws also deserve interest for the novelty of the formalization of their DSR-relativistic symmetries. It is rather well understood that in cases like $\kappa$-dS the momentum space is a group manifold, and the formalization of the DSR-relativistic symmetries can be based on the mathematics of some corresponding Hopf algebras, then leading inevitably to non-associative but commutative composition laws. It would be important to understand what sort of general mathematical structures should replace Hopf algebras in cases where the momentum space is DSR-relativistic but the composition law is commutative but non-associative.

Perhaps the most fascinating issue we here contemplated concerns the general possibility of associating firmly a momentum-space geometry to a given setup for Planck-scale-deformed relativistic kinematics. We here established that the possible choices of laws of composition of momenta are more numerous than the possible choices of affine connection on a momentum space. This inevitably raises the issue of finding a most natural way for introducing a link between affine connection and form of the composition law. As a first contribution toward tackling this issue we here offered several observations concerning the differences between the standard geometric-interpretation prescription, here coded in Eq. 6), and the novel geometric interpretation we introduced through Eqs. (13)-(15).

\section{Appendix A: Decomposition of the Connection}

A well known result of differential geometry states that, given a connection $\Gamma$ and a metric $g$, the connection can be decomposed as:

$$
\Gamma_{\nu}^{\lambda \mu}=A_{\nu}^{\lambda \mu}+K_{\nu}^{\lambda \mu}+V_{\nu}^{\lambda \mu}
$$

$A^{\lambda \mu}{ }_{\nu}$ is the Levi-Civita connection, defined by the requirement that the covariant derivative associated to it vanishes when applied to the metric tensor: 


$$
\left\{\begin{array}{l}
\nabla_{(A)}^{\lambda} g^{\mu \nu}=\partial^{\lambda} g^{\mu \nu}-A^{\lambda \mu}{ }_{\theta} g^{\theta \nu}-A^{\lambda \nu}{ }_{\theta} g^{\theta \mu}=0 \\
A_{\nu}^{\lambda \mu}=A_{\nu}^{\mu \lambda}
\end{array}\right.
$$

This system is solved by:

$$
A_{\nu}^{\lambda \mu}=\frac{1}{2} g_{\nu \theta}\left(\partial^{\mu} g^{\theta \lambda}+\partial^{\lambda} g^{\theta \mu}-\partial^{\theta} g^{\lambda \mu}\right)
$$

$K^{\lambda \mu}{ }_{\nu}$ is called the contortion tensor and is defined by:

$$
K^{\lambda \mu}{ }_{\theta} g^{\theta \nu}+K^{\lambda \nu}{ }_{\theta}^{\theta \mu}=0
$$

while $V^{\lambda \mu}{ }_{\nu}$ is the cononmetricity, defined by:

$$
\nabla_{(\Gamma)}^{\lambda} g^{\mu \nu}=-V^{\lambda \mu} g^{\theta \nu}-V^{\lambda \nu}{ }_{\theta}^{\theta \mu}
$$

where $\nabla_{(\Gamma)}$ is the covariant derivative associated to the connection $\Gamma$.

In order to express $K$ and $V$ in terms of the metric $g$ and the connection $\Gamma$ it is convenient to make use of the torsion tensor and the nonmetricity tensor, defined respectively as:

$$
\left\{\begin{array}{l}
T_{\nu \mu}^{\lambda \mu}=2 \Gamma^{[\lambda \mu]}{ }_{\nu} \\
Q^{\lambda \mu \nu}=\nabla^{\nu} g^{\lambda \mu}
\end{array}\right.
$$

Observing that:

$$
\left(\partial^{\lambda} g^{\mu \nu}-\partial^{\nu} g^{\lambda \mu}-\partial^{\mu} g^{\nu \lambda}\right)-2 \Gamma^{[\lambda \mu]}{ }_{\theta} g^{\theta \nu}-2 \Gamma^{[\lambda \nu]}{ }_{\theta} g^{\theta \mu}+2 \Gamma^{(\mu \nu)}{ }_{\theta} g^{\theta \lambda}=Q^{\mu \nu \lambda}-Q^{\lambda \mu \nu}-Q^{\nu \lambda \mu}
$$

and using the definition of the torsion tensor, one finds:

$$
\Gamma^{\mu \nu \lambda}=A^{\mu \nu \lambda}+\frac{1}{2}\left(T^{\mu \nu \lambda}+T^{\lambda \mu \nu}-T^{\nu \lambda \mu}\right)+\frac{1}{2}\left(Q^{\mu \nu \lambda}-Q^{\lambda \mu \nu}-Q^{\nu \lambda \mu}\right)
$$

Finally, lowering one index:

$$
\Gamma_{\lambda}^{\mu \nu}=A_{\lambda}^{\mu \nu}+\frac{1}{2}\left(T_{\lambda}^{\mu \nu}+T_{\lambda}^{\mu \nu}-T_{\lambda}^{\nu}{ }_{\lambda}^{\mu}\right)+\frac{1}{2}\left(Q_{\lambda}^{\mu \nu}-Q_{\lambda}{ }^{\mu \nu}-Q^{\nu}{ }_{\lambda}{ }^{\mu}\right)
$$

The second and the third term in the above expression satisfy the defining properties of, respectively, $K$ and $V$. Therefore we define:

$$
\left\{\begin{array}{l}
K_{\lambda}^{\mu \nu}=\frac{1}{2}\left(T^{\mu \nu}{ }_{\lambda}+T_{\lambda}{ }^{\mu \nu}-T^{\nu}{ }_{\lambda}{ }^{\mu}\right) \\
V_{\lambda}^{\mu \nu}=\frac{1}{2}\left(Q_{\lambda}^{\mu \nu}{ }_{\lambda}-Q_{\lambda}{ }^{\mu \nu}-Q^{\nu}{ }_{\lambda}{ }^{\mu}\right)
\end{array}\right.
$$

From these definitions one easily verifies that:

$$
\left\{\begin{array}{l}
K^{\mu(\nu \lambda)}=\frac{1}{2}\left(T^{\mu(\nu \lambda)}+T^{(\lambda \mu \nu)}\right)=0 \\
V^{[\mu \nu] \lambda}=0=Q^{[\mu \nu] \lambda}
\end{array}\right.
$$

Therefore, the symmetries of the contortion do not involve the differential index, while the symmetries of the cononmetricity do, as is the case for the Levi-Civita connection. 


\section{Appendix B: Compatibility conditions for the invariance of the distance function}

We want to find the symmetries of the distance function

$$
d_{\ell}^{2}(k, q)=\int d t \sqrt{g^{\mu \nu}(\gamma(t)) \dot{\gamma}_{\mu}(t) \dot{\gamma}_{\nu}(t)}
$$

where $\gamma(t)$ is the metric geodesic connecting the points $q$ and $k$.

So we look for the set of infinitesimal transformations on momentum space $p_{\mu} \rightarrow \mathcal{S}_{\mu}^{(\ell)}(p)=p_{\mu}+\mathcal{T}_{\mu}^{(\ell)}(p)$ which act on the two points $q$ and $k$ preserving the geodesic distance between them:

$$
d_{\ell}^{2}\left(k+\mathcal{T}^{(\ell)}(k), q+\mathcal{T}^{(\ell)}(q)\right)=d_{\ell}^{2}(k, q)
$$

Thanks to the monotone behavior of the square root we can equivalently ask that the following quantity is left unchanged by the transformations:

$$
\tilde{d}_{\ell}(k, q)=\int d t\left(g^{\mu \nu}(\gamma(t)) \dot{\gamma}_{\mu}(t) \dot{\gamma}_{\nu}(t)\right)
$$

Defining $L(\gamma, \dot{\gamma}) \equiv g^{\mu \nu}(\gamma) \dot{\gamma}_{\mu} \dot{\gamma}_{\nu}$, the variation of (B3) takes the form:

$$
\delta \int d t L(\gamma(t), \dot{\gamma}(t))=\int\left(\frac{\partial L}{\partial \gamma}-\frac{d}{d t}\left(\frac{\partial L}{\partial \dot{\gamma}}\right)\right) \delta \gamma d t+\left.\left(\frac{\partial L}{\partial \dot{\gamma}} \delta \gamma\right)\right|_{t=0} ^{t=1}
$$

where $\delta \gamma(t)$ is such that $\gamma(t)+\delta \gamma(t)$ is the geodesic connecting $q+\mathcal{T}(q)$ and $k+\mathcal{T}(k)$, which must satisfy, in particular, $\delta \gamma(0)=\mathcal{T}(q)$ and $\delta \gamma(1)=\mathcal{T}(k)$.

The argument of the integral on the right-hand side of $B 4$ is nothing but the geodesic equation which is solved, by definition, by the curve $\gamma$. So this contribution to the variation of the distance function vanishes and we only need to ask for the boundary term to vanish:

$$
\left.\left(\frac{\partial L}{\partial \dot{\gamma}} \delta \gamma\right)\right|_{t=0} ^{t=1}=0
$$

In order to solve (B5) the first step is to note that, since we have to solve $(\mathrm{B} 5)$ for any couple of points $q$ and $k$, we can equivalently solve:

$$
\frac{d}{d t}\left(\frac{\partial L}{\partial \dot{\gamma}} \delta \gamma\right)=0
$$

This implies that the Killing equation must be satisfied by $\delta \gamma$ :

$$
\begin{gathered}
\frac{d}{d t}\left(\frac{\partial L}{\partial \dot{\gamma}} \delta \gamma\right)=\left(\partial^{\theta} g^{\alpha \nu} \delta \gamma_{\alpha}-g^{\alpha \eta} A_{\eta}^{\theta \nu} \delta \gamma_{\alpha}+g^{\alpha \nu} \partial^{\theta} \delta \gamma_{\alpha}\right) \dot{\gamma}_{\theta} \dot{\gamma}_{\nu} \\
\Rightarrow 0=\left(\partial^{(\theta} g^{\alpha \nu)} \delta \gamma_{\alpha}-g^{\alpha \eta} A_{\eta}^{\theta \nu} \delta \gamma_{\alpha}+g^{\alpha(\nu} \partial^{\theta)} \delta \gamma_{\alpha}\right)=\left(\partial^{(\theta} \delta \gamma^{\nu)}-A_{\eta}^{\theta \nu} \delta \gamma^{\eta}\right)
\end{gathered}
$$

that is:

$$
\nabla_{A}^{(\theta} \delta \gamma^{\nu)}=0
$$

By identifying $\delta \gamma$ with $\mathcal{T}^{(\ell)} 6$ we get the condition on the infinitesimal transformations $\mathcal{T}$ ensuring the invariance of the distance function:

\footnotetext{
${ }^{6}$ It is possible to show that this identification is a necessary condition in order for $\mathrm{B} 4$ to hold.
} 


$$
\nabla_{A}^{(\theta} \mathcal{T}^{(\ell) \nu)}=0
$$

We now turn to the task of finding the explicit form of the symmetries $\mathcal{T}^{(\ell)}$ which preserve the distance function associated to the de Sitter metric.

Using the de Sitter Levi-Civita connection

$$
A^{\lambda \mu}{ }_{\nu}(p)=2 \ell \delta_{0}^{(\lambda} \delta_{1}^{\mu)} \delta_{\nu}^{1}+\ell \delta_{1}^{\lambda} \delta_{1}^{\mu} \delta_{\nu}^{0} e^{\ell p_{0}}
$$

the condition $(\mathrm{B} 9)$ becomes

$$
\left\{\begin{array}{l}
\partial^{0} \mathcal{T}^{(\ell) 0}=0 \\
\frac{1}{2}\left(\partial^{0} \mathcal{T}^{(\ell) 1}+\partial^{1} \mathcal{T}^{(\ell) 0}\right)-\ell \mathcal{T}^{(\ell) 1}=0 \\
\partial^{1} \mathcal{T}^{(\ell) 1}-\ell e^{\ell p_{0}} \mathcal{T}^{(\ell) 0}=0
\end{array}\right.
$$

and has the solution:

$$
\left\{\begin{array}{l}
\mathcal{T}_{0}^{(\ell)}(p)=\xi p_{1}+\gamma \\
\mathcal{T}_{1}^{(\ell)}(p)=-\frac{\xi}{2 \ell} e^{-2 \ell p_{0}}-\frac{\ell}{2} \xi p_{1}^{2}-\ell \gamma p_{1}+\beta
\end{array}\right.
$$

where $\xi, \beta$ and $\gamma$ are constants of integration.

By requiring that in the limit $\ell \rightarrow 0$ we recover the Poincaré group of trasformation we redefine: $\beta \rightarrow \frac{\xi}{2 \ell}+\beta$, obtaining:

$$
\left\{\begin{array}{l}
\mathcal{T}_{0}^{(\ell)}(p)=\xi p_{1}+\gamma \\
\mathcal{T}_{1}^{(\ell)}(p)=\frac{\xi}{2 \ell}\left(1-e^{-2 \ell p_{0}}\right)-\xi \frac{\ell}{2} p_{1}^{2}-\gamma \ell p_{1}+\beta
\end{array}\right.
$$

Therefore we get three killing vectors associated to the three constants of integration. In particular we recognize a deformation of the translational sector in the algebra associated to $\beta$ and $\gamma$, while the deformation of the Lorentz sector $\left(\left.\mathcal{N}^{(\ell)} \equiv \mathcal{T}^{(\ell)}\right|_{\text {Lorentz }}\right.$ that solves $\left.\mathcal{N}^{(\ell)}(0)=0\right)$ is:

$$
\left\{\begin{array}{l}
\mathcal{N}_{0}^{(\ell)}(p)=\xi p_{1} \\
\mathcal{N}_{1}^{(\ell)}(p)=\frac{\xi}{2 \ell}\left(1-e^{-2 \ell p_{0}}\right)-\xi \frac{\ell}{2} p_{1}^{2}
\end{array}\right.
$$

In particular, to the second order in $\ell$ one finds:

$$
\left\{\begin{array}{l}
\mathcal{N}_{0}^{(\ell)}=\xi p_{1} \\
\mathcal{N}_{1}^{(\ell)}(p)=\xi p_{0}-\xi \ell\left(p_{0}^{2}+\frac{1}{2} p_{1}^{2}\right)+\frac{2}{3} \xi \ell^{2} p_{0}^{3}
\end{array}\right.
$$

\section{Appendix C: Second Order Composition Law}

We want to develop the form of the composition law associated to some given connection $\Gamma$ at second order in the Planck length $\ell$.

Our starting point is the definition of the composition law associated to a given connection, which we note down here again:

$$
\left\{\begin{array}{l}
\frac{d}{d t} \frac{d}{d s} \gamma_{\lambda}(s, t)+\Gamma_{\lambda}^{\alpha \beta}\left(\zeta^{(s)}(t)\right) \frac{d \zeta_{\alpha}^{(s)}(t)}{d t} \frac{d \gamma_{\beta}(s, t)}{d s}=0 \\
\gamma_{\lambda}(s, 0)=\gamma_{\lambda}^{(k)}(s) \\
\gamma_{\lambda}(0, t)=\gamma_{\lambda}^{(q)}(t) \\
\gamma_{\lambda}(1,1)=\left(q \oplus_{\ell} k\right)_{\lambda}
\end{array}\right.
$$


where $\zeta$ is a geodesic defined by:

$$
\left\{\begin{array}{l}
\ddot{\zeta}_{\lambda}^{(s)}(t)+\Gamma_{\lambda}^{\alpha \beta} \dot{\zeta}_{\alpha}^{(s)}(t) \dot{\zeta}_{\beta}^{(s)}(t)=0 \\
\zeta_{\lambda}^{(s)}(0)=\gamma_{\lambda}(s, 0)=\gamma_{\lambda}^{(k)}(s) \\
\zeta_{\lambda}^{(s)}(1)=\gamma_{\lambda}(s, 1)
\end{array}\right.
$$

The geodesics $\zeta^{(s)}, \gamma^{(k)}$ and $\gamma^{(q)}$ are all connection geodesics which reduce to metric geodesics when the connection $\Gamma$ has only the Levi-Civita contribution $A$.

The second order solution is found by expanding the connection as follows:

$$
\Gamma_{\nu}^{\lambda \mu}\left(\zeta^{(s)}(t)\right)=\Gamma_{\nu}^{\lambda \mu}(0)+\left.\partial^{\theta} \Gamma_{\nu}^{\lambda \mu}\right|_{\zeta=0} \zeta_{\theta}^{(s)}(t)
$$

where the $\zeta^{(s)}(t)$ on the right hand side has to be taken at the zeroth order. In the following, in order to unburden the notation we will use :

$$
\left\{\begin{array}{l}
\Gamma_{\nu}^{\lambda \mu}{ }_{\nu}(0) \equiv \tilde{\Gamma}^{\lambda \mu}{ }_{\nu} \\
\left.\partial^{\eta} \Gamma^{\lambda \mu}\right|_{\zeta=0} \equiv \partial^{\eta} \tilde{\Gamma}^{\lambda \mu}{ }_{\nu}
\end{array}\right.
$$

Note that this notation is slightly different from the one used in the main text of the paper in section III

Let us now compute the composition law defined by the system of equations (C1) and (C2).

At the zeroth order

$$
\left\{\begin{array}{l}
\frac{d}{d t} \frac{d}{d s} \gamma_{\lambda}(s, t)=0 \\
\ddot{\zeta}_{\lambda}^{(s)}(t)=0
\end{array}\right.
$$

which, taking into account the boundary conditions, have solutions:

$$
\left\{\begin{array}{l}
\gamma_{\lambda}(s, t)=q_{\lambda} t+k_{\lambda} s \\
\zeta_{\lambda}^{(s)}(t)=q_{\lambda} t+k_{\lambda} s
\end{array}\right.
$$

So up to the zeroth order the composition law is:

$$
\left(q \oplus_{\ell} k\right)_{\lambda}=q_{\lambda}+k_{\lambda}
$$

At the first order in $\ell$ the differential equation in $\mathrm{C} 1$ reads:

$$
\frac{d}{d t} \frac{d}{d s} \gamma_{\lambda}(s, t)+\tilde{\Gamma}_{\lambda}^{\alpha \beta}{\frac{d \zeta_{\alpha}^{(s)}(t)}{d t}}_{\frac{d \gamma_{\beta}(s, t)}{d s}}=0
$$

where $\frac{d \zeta_{\alpha}^{(s)}(t)}{d t}$ and $\frac{d \gamma_{\beta}(s, t)}{d s}$ have to be taken at zeroth order, so that the equation takes the form:

$$
\frac{d}{d t} \frac{d}{d s} \gamma_{\lambda}(s, t)+\tilde{\Gamma}_{\lambda}^{\alpha \beta} q_{\alpha} k_{\beta}=0
$$

Solving for $\gamma_{\lambda}(s, t)$ and taking into account the boundary conditions we find that up to first order:

$$
\gamma_{\lambda}(s, t)=\gamma_{\lambda}^{(q)}(t)+\gamma_{\lambda}^{(k)}(s)-\tilde{\Gamma}_{\lambda}^{\alpha \beta} q_{\alpha} k_{\beta} s t
$$

so that the first-order composition law takes the form:

$$
\left(q \oplus_{\ell} k\right)_{\lambda}=q_{\lambda}+k_{\lambda}-\tilde{\Gamma}_{\lambda}^{\alpha \beta} q_{\alpha} k_{\beta}
$$

For what concerns the first-order description of the geodesic $\zeta^{(s)}(t)$ we have that it must satisfy the following differential equation:

$$
\ddot{\zeta}_{\lambda}^{(s)}+\tilde{\Gamma}_{\lambda}^{\alpha \beta} \dot{\zeta}_{\alpha}^{(s)} \dot{\zeta}_{\beta}^{(s)}=0
$$

which, together with the boundary conditions, has solution: 


$$
\zeta_{\lambda}^{(s)}(t)=\gamma_{\lambda}^{(k)}(s)+\gamma_{\lambda}^{(q)}(t)-\tilde{\Gamma}_{\lambda \beta}^{\alpha \beta} q_{\alpha} k_{\beta} s t
$$

The second-order contribution to the differential equation in $\mathrm{C} 1$ is:

$$
\begin{aligned}
& \left(\frac{d}{d t} \frac{d}{d s} \gamma_{\lambda}(s, t)\right)^{(2)}+\tilde{\Gamma}_{\lambda}^{\alpha \beta}\left(\frac{d \zeta_{\alpha}^{(s)}(t)}{d t}\right)^{(0)}\left(\frac{d \gamma_{\beta}(s, t)}{d s}\right)^{(1)}+\tilde{\Gamma}_{\lambda}^{\alpha \beta}\left(\frac{d \zeta_{\alpha}^{(s)}(t)}{d t}\right)^{(1)}\left(\frac{d \gamma_{\beta}(s, t)}{d s}\right)^{(0)} \\
& -\tilde{\Gamma}_{\lambda}^{\alpha \beta}\left(\frac{d \zeta_{\alpha}^{(s)}(t)}{d t}\right)^{(0)}\left(\frac{d \gamma_{\beta}(s, t)}{d s}\right)^{(0)}+\partial^{\theta} \tilde{\Gamma}_{\lambda{ }_{\lambda} \zeta_{\theta}}\left(\frac{d \zeta_{\alpha}^{(s)}(t)}{d t}\right)^{(0)}\left(\frac{d \gamma_{\beta}(s, t)}{d s}\right)^{(0)}=0
\end{aligned}
$$

where the superscripts refer to the $\ell$ order to be considered for each term.

Substituting the zeroth order results and integrating one finds:

$$
\begin{aligned}
\gamma_{\lambda}(s, t)= & B_{\lambda}(t)+\int d s^{\prime} A_{\lambda}(s)-\tilde{\Gamma}^{\alpha \beta}{ }_{\lambda} q_{\alpha} \int d t^{\prime} \int d s^{\prime}\left(\frac{d \gamma_{\beta}}{d s}\right)^{(1)}-\tilde{\Gamma}_{\lambda}^{\alpha \beta} \int d s^{\prime} \int d t^{\prime}\left(\frac{d \zeta_{\alpha}}{d t}\right)^{(1)} k_{\beta}+ \\
& +\tilde{\Gamma}^{\alpha \beta}{ }_{\lambda} q_{\alpha} k_{\beta} s t-\frac{1}{2} \partial^{\theta} \tilde{\Gamma}^{\alpha \beta}{ }_{\lambda}\left(q_{\theta} s t^{2}+k_{\theta} s^{2} t\right) q_{\alpha} k_{\beta}
\end{aligned}
$$

Then observing that

$$
\left\{\begin{array}{l}
\int d s^{\prime}\left(\frac{d \gamma_{\beta}}{d s}\right)^{(1)}=\gamma_{\beta}^{(k)}(s)-\tilde{\Gamma}^{\gamma \delta}{ }_{\beta} q_{\gamma} k_{\delta} s t \\
\int d t^{\prime}\left(\frac{d \zeta_{\alpha}^{s}}{d t}\right)^{(1)}=\gamma_{\alpha}^{(q)}(t)-\tilde{\Gamma}^{\gamma \delta}{ }_{\alpha} q_{\gamma} k_{\delta} s t
\end{array}\right.
$$

and using the boundary conditions one obtains that up to second order

$$
\begin{aligned}
\gamma_{\lambda}(s, t)= & \gamma_{\lambda}^{(q)}(t)+\gamma_{\lambda}^{(k)}(s)-\tilde{\Gamma}_{\lambda}^{\alpha \beta}{ }_{\lambda} q_{\alpha}\left(\left(\gamma_{\beta}^{(k)}(s)\right)^{(1)} t-\frac{1}{2} \tilde{\Gamma}_{\beta}^{\gamma \delta}{ }_{\beta} q_{\gamma} k_{\delta} s t^{2}\right)+ \\
& -\tilde{\Gamma}_{\lambda}^{\alpha \beta}\left(\left(\gamma_{\alpha}^{(q)}(t)\right)^{(1)} s-\frac{1}{2} \tilde{\Gamma}_{\alpha}^{\gamma \delta}{ }_{\alpha} q_{\gamma} k_{\delta} s^{2} t\right) k_{\beta}+\tilde{\Gamma}_{\lambda}^{\alpha \beta}{ }_{\lambda} q_{\alpha} k_{\beta} s t-\frac{1}{2} \partial^{\theta} \tilde{\Gamma}_{\lambda}^{\alpha \beta}\left(q_{\theta} s t^{2}+k_{\theta} s^{2} t\right) q_{\alpha} k_{\beta}
\end{aligned}
$$

So up to second order the composition law $q \oplus_{\ell} k$ associated to a connection $\Gamma$ takes the form:

$$
\left(q \oplus_{\ell} k\right)_{\lambda}=q_{\lambda}+k_{\lambda}-\tilde{\Gamma}_{\lambda}^{\alpha \beta}{ }_{\lambda} q_{\alpha} k_{\beta}+\frac{1}{2} \tilde{\Gamma}_{\lambda}^{\alpha \beta} \tilde{\Gamma}_{\beta}^{\gamma \delta} q_{\alpha} q_{\gamma} k_{\delta}+\frac{1}{2} \tilde{\Gamma}_{\lambda}^{\alpha \beta} \tilde{\Gamma}_{\alpha}^{\gamma \delta} q_{\gamma} k_{\beta} k_{\delta}-\frac{1}{2} \partial^{\theta} \tilde{\Gamma}_{\lambda}^{\alpha \beta}{ }_{\lambda}\left(q_{\theta}+k_{\theta}\right) q_{\alpha} k_{\beta}
$$

If we use, for example, the Levi-Civita connection associated to the de Sitter metric

$$
\Gamma_{\nu}^{\lambda \mu}(p)=\ell\left(\delta_{0}^{\lambda} \delta_{1}^{\mu}+\delta_{0}^{\mu} \delta_{1}^{\lambda}\right) \delta_{\nu}^{1}+\ell \delta_{1}^{\lambda} \delta_{1}^{\mu} \delta_{\nu}^{0} e^{\ell p_{0}}
$$

we get the second order composition law for "proper de Sitter" :

$$
\left\{\begin{array}{l}
\left(q \oplus_{\ell} k\right)_{0}=q_{0}+k_{0}-\ell q_{1} k_{1}+\frac{\ell^{2}}{2}\left[-q_{1} k_{1}\left(q_{0}+k_{0}\right)+q_{0} k_{1}^{2}+q_{1}^{2} k_{0}\right] \\
\left(q \oplus_{\ell} k\right)_{1}=q_{1}+k_{1}-\ell\left(q_{0} k_{1}+q_{1} k_{0}\right)+\frac{\ell^{2}}{2}\left[\left(q_{0} k_{1}+q_{1} k_{0}\right)\left(q_{0}+k_{0}\right)+q_{1} k_{1}^{2}+q_{1}^{2} k_{1}\right]
\end{array}\right.
$$

\section{Appendix D: Second Order Translated Composition Law}

The translated composition law associated to the subtraction point $p$ was defined in equations (18) and (19), reported here for convenience:

$$
\left\{\begin{array}{l}
\frac{d}{d t} \frac{d}{d s} \gamma_{\lambda}^{[p]}(s, t)+\Gamma^{\alpha \beta}{ }_{\lambda}\left(\zeta^{(s)}(t)\right) \frac{d \zeta_{\alpha}^{(s)}(t)}{d t} \frac{d \gamma_{\beta}^{[p]}(s, t)}{d s}=0 \\
\gamma_{\lambda}^{[p]}(s, 0)=\gamma_{\lambda}^{(k, p)}(s) \\
\gamma_{\lambda}^{[p]}(0, t)=\gamma_{\lambda}^{(q, p)}(t) \\
\gamma_{\lambda}^{[p]}(1,1)=\left(q \oplus_{\ell}^{[p]} k\right)_{\lambda}
\end{array}\right.
$$




$$
\left\{\begin{array}{l}
\ddot{\zeta}_{\lambda}^{(s)}(t)+\Gamma_{\lambda}^{\alpha \beta}\left(\zeta^{(s)}(t)\right) \dot{\zeta}_{\alpha}^{(s)}(t) \dot{\zeta}_{\beta}^{(s)}(t)=0 \\
\zeta_{\lambda}^{(s)}(0)=\gamma_{\lambda}^{(k, p)}(s) \\
\zeta_{\lambda}^{(s)}(1)=\gamma_{\lambda}^{[p]}(s, 1)
\end{array}\right.
$$

and

$$
\left\{\begin{array}{l}
\frac{d^{2}}{d t^{2}} \gamma_{\lambda}^{(q, p)}(t)+\Gamma_{\lambda}^{\mu \nu}\left(\gamma^{(q, p)}(t)\right) \frac{d \gamma_{\mu}^{(q, p)}(t)}{d t} \frac{d \gamma_{\nu}^{(q, p)}(t)}{d t}=0 \\
\gamma^{(q, p)}(0)=p \\
\gamma^{(q, p)}(1)=q
\end{array}\right.
$$

with a similar formula holding also for $\gamma^{(k, p)}$.

In the following we proceed along the lines of the previous section to derive the second order expansion of the translated composition law.

At the zeroth order:

$$
\left\{\begin{array}{l}
\frac{d}{d t} \frac{d}{d s} \gamma_{\lambda}^{[p]}(s, t)=0 \\
\ddot{\zeta}_{\lambda}^{(s)}(t)=0
\end{array}\right.
$$

Taking into account the boundary conditions these have solution:

$$
\left\{\begin{array}{l}
\gamma_{\lambda}^{[p]}(s, t)=\gamma_{\lambda}^{(k, p)}(s)+\gamma_{\lambda}^{q, p}(t)-p_{\lambda}=p_{\lambda}+\left(k_{\lambda}-p_{\lambda}\right) s+\left(q_{\lambda}-p_{\lambda}\right) t \\
\zeta_{\lambda}^{(s)}(t)=p_{\lambda}+\left(k_{\lambda}-p_{\lambda}\right) s+\left(q_{\lambda}-p_{\lambda}\right) t
\end{array}\right.
$$

So up to the zeroth order the composition law is:

$$
\left(q \oplus_{\ell}^{[p]} k\right)_{\lambda}=q_{\lambda}+k_{\lambda}-p_{\lambda}
$$

The first-order expansion of the differential equation in (D1) is

$$
\frac{d}{d t} \frac{d}{d s} \gamma_{\lambda}^{[p]}(s, t)+\tilde{\Gamma}_{\lambda}^{\alpha \beta} \frac{d \zeta_{\alpha}^{(s)}(t)}{d t} \frac{d \gamma_{\beta}^{[p]}(s, t)}{d s}=0
$$

where $\frac{d \zeta_{\alpha}^{(s)}(t)}{d t}$ and $\frac{d \gamma_{\beta}^{[p]}(s, t)}{d s}$ have to be evaluated at the zeroth order, so that the equation we have to solve is:

$$
\frac{d}{d t} \frac{d}{d s} \gamma_{\lambda}^{[p]}(s, t)+\tilde{\Gamma}_{\lambda}^{\alpha \beta}\left(q_{\alpha}-p_{\alpha}\right)\left(k_{\beta}-p_{\beta}\right)=0
$$

Solving for $\gamma_{\lambda}^{[p]}(s, t)$ and taking into account the boundary conditions we find that up to first order:

$$
\gamma_{\lambda}^{[p]}(s, t)=\gamma_{\lambda}^{(q, p)}(t)+\gamma_{\lambda}^{(k, p)}(s)-p_{\lambda}-\tilde{\Gamma}_{\lambda}^{\alpha \beta}\left(q_{\alpha}-p_{\alpha}\right)\left(k_{\beta}-p_{\beta}\right) s t
$$

so that the first-order composition law takes the form:

$$
\left(q \oplus_{\ell}^{[p]} k\right)_{\lambda}=q_{\lambda}+k_{\lambda}-p_{\lambda}-\tilde{\Gamma}_{\lambda}^{\alpha \beta}\left(q_{\alpha}-p_{\alpha}\right)\left(k_{\beta}-p_{\beta}\right)
$$

For what concerns the first-order description of the geodesic $\zeta^{(s)}(t)$ we have that it must satisfy the following differential equation:

$$
\ddot{\zeta}_{\lambda}^{(s)}+\tilde{\Gamma}_{\lambda}^{\alpha \beta} \dot{\zeta}_{\alpha}^{(s)} \dot{\zeta}_{\beta}^{(s)}=0
$$

which, together with the boundary conditions, has solution:

$$
\zeta_{\lambda}^{(s)}(t)=\gamma_{\lambda}^{(k, p)}(s)+\gamma_{\lambda}^{(q, p)}(t)-p_{\lambda}-\tilde{\Gamma}_{\lambda}^{\alpha \beta}\left(q_{\alpha}-p_{\alpha}\right)\left(k_{\beta}-p_{\beta}\right) s t
$$


The second-order contribution to the differential equation in (D1) is:

$$
\begin{aligned}
& \left(\frac{d}{d t} \frac{d}{d s} \gamma_{\lambda}^{[p]}(s, t)\right)^{(2)}+\tilde{\Gamma}_{\lambda}^{\alpha \beta}\left(\frac{d \zeta_{\alpha}^{(s)}(t)}{d t}\right)^{(0)}\left(\frac{d \gamma_{\beta}^{[p]}(s, t)}{d s}\right)^{(1)}+\tilde{\Gamma}_{\lambda}^{\alpha \beta}\left(\frac{d \zeta_{\alpha}^{(s)}(t)}{d t}\right)^{(1)}\left(\frac{d \gamma_{\beta}^{[p]}(s, t)}{d s}\right)^{(0)} \\
& -\tilde{\Gamma}_{\lambda}^{\alpha \beta}\left(\frac{d \zeta_{\alpha}^{(s)}(t)}{d t}\right)^{(0)}\left(\frac{d \gamma_{\beta}^{[p]}(s, t)}{d s}\right)^{(0)}+\partial^{\theta} \tilde{\Gamma}_{\lambda}^{\alpha \beta} \zeta_{\theta}\left(\frac{d \zeta_{\alpha}^{(s)}(t)}{d t}\right)^{(0)}\left(\frac{d \gamma_{\beta}^{[p]}(s, t)}{d s}\right)^{(0)}=0
\end{aligned}
$$

where the superscripts refer to the $\ell$ order to be considered for each term.

Substituting the zeroth order results and integrating one finds:

$$
\begin{aligned}
\gamma_{\lambda}^{[p]}(s, t)= & B_{\lambda}(t)+\int d s^{\prime} A_{\lambda}(s)-\tilde{\Gamma}^{\alpha \beta}{ }_{\lambda}\left(q_{\alpha}-p_{\alpha}\right) \int d t^{\prime} \int d s^{\prime}\left(\frac{d \gamma_{\beta}^{[p]}}{d s}\right)^{(1)}-\tilde{\Gamma}^{\alpha \beta}{ }_{\lambda} \int d s^{\prime} \int d t^{\prime}\left(\frac{d \zeta_{\alpha}}{d t}\right)^{(1)}\left(k_{\beta}-p_{\beta}\right)+ \\
& +\tilde{\Gamma}^{\alpha \beta}{ }_{\lambda}\left(q_{\alpha}-p_{\alpha}\right)\left(k_{\beta}-p_{\beta}\right) s t-\frac{1}{2} \partial^{\theta} \tilde{\Gamma}^{\alpha \beta}{ }_{\lambda}\left(\left(q_{\theta}-p_{\theta}\right) s t^{2}+\left(k_{\theta}-p_{\theta}\right) s^{2} t+2 p_{\theta} s t\right)\left(q_{\alpha}-p_{\alpha}\right)\left(k_{\beta}-p_{\beta}\right)
\end{aligned}
$$

Then observing that

$$
\left\{\begin{array}{l}
\int d s^{\prime}\left(\frac{d \gamma_{\beta}}{d s}\right)^{(1)}=\gamma_{\beta}^{(k, p)}(s)-p_{\beta}-\tilde{\Gamma}_{\beta}^{\gamma \delta}\left(q_{\gamma}-p_{\gamma}\right)\left(k_{\delta}-p_{\delta}\right) s t \\
\int d t^{\prime}\left(\frac{d \zeta_{\alpha}^{s}}{d t}\right)^{(1)}=\gamma_{\alpha}^{(q, p)}(t)-p_{\alpha}-\tilde{\Gamma}_{\alpha}^{\gamma \delta}\left(q_{\gamma}-p_{\gamma}\right)\left(k_{\delta}-p_{\delta}\right) s t
\end{array}\right.
$$

and using the boundary conditions one obtains that up to second order

$$
\begin{aligned}
\gamma_{\lambda}(s, t)= & \gamma_{\lambda}^{(q, p)}(t)+\gamma_{\lambda}^{(k, p)}(s)-p_{\lambda}-\tilde{\Gamma}_{\lambda}^{\alpha \beta}\left(q_{\alpha}-p_{\alpha}\right)\left(\left(\gamma_{\beta}^{(k, p)}(s)\right)^{(1)} t-p_{\beta} t-\frac{1}{2} \tilde{\Gamma}_{\beta}^{\gamma \delta}\left(q_{\gamma}-p_{\gamma}\right)\left(k_{\delta}-p_{\delta}\right) s t^{2}\right)+ \\
& -\tilde{\Gamma}^{\alpha \beta}{ }_{\lambda}\left(\left(\gamma_{\alpha}^{(q, p)}(t)\right)^{(1)} s-p_{\alpha} s-\frac{1}{2} \tilde{\Gamma}_{\alpha \delta}^{\gamma \delta}\left(q_{\gamma}-p_{\gamma}\right)\left(k_{\delta}-p_{\delta}\right) s^{2} t\right)\left(k_{\beta}-p_{\beta}\right)+\tilde{\Gamma}^{\alpha \beta}{ }_{\lambda}\left(q_{\alpha}-p_{\alpha}\right)\left(k_{\beta}-p_{\beta}\right) s t \\
& -\frac{1}{2} \partial^{\theta} \tilde{\Gamma}_{\lambda}^{\alpha \beta}\left(2 p_{\theta} s t+\left(q_{\theta}-p_{\theta}\right) s t^{2}+\left(k_{\theta}-p_{\theta}\right) s^{2} t\right)\left(q_{\alpha}-p_{\alpha}\right)\left(k_{\beta}-p_{\beta}\right)
\end{aligned}
$$

So up to second order the translated composition law $q \oplus_{\ell}^{[p]} k$ associated to a connection $\Gamma$ takes the form:

$$
\begin{aligned}
\left(q \oplus_{\ell}^{[p]} k\right)_{\lambda}= & q_{\lambda}+k_{\lambda}-p_{\lambda}-\tilde{\Gamma}^{\alpha \beta}{ }_{\lambda}\left(q_{\alpha}-p_{\alpha}\right)\left(k_{\beta}-p_{\beta}\right)+\frac{1}{2} \tilde{\Gamma}^{\alpha \beta}{ }_{\lambda} \tilde{\Gamma}^{\gamma \delta}{ }_{\beta}\left(q_{\alpha}-p_{\alpha}\right)\left(q_{\gamma}-p_{\gamma}\right)\left(k_{\delta}-p_{\delta}\right) \\
& +\frac{1}{2} \tilde{\Gamma}^{\alpha \beta}{ }_{\lambda} \tilde{\Gamma}^{\gamma \delta}{ }_{\alpha}\left(q_{\gamma}-p_{\gamma}\right)\left(k_{\delta}-p_{\delta}\right)\left(k_{\beta}-p_{\beta}\right)-\frac{1}{2} \partial^{\theta} \tilde{\Gamma}^{\alpha \beta}{ }_{\lambda}\left(q_{\theta}+k_{\theta}\right)\left(q_{\alpha}-p_{\alpha}\right)\left(k_{\beta}-p_{\beta}\right)
\end{aligned}
$$

Let us now verify that the connection can be derived from the translated composition law by using:

$$
\Gamma_{\lambda}^{\mu \nu}(p)=-\left.\frac{\partial}{\partial q_{\mu}} \frac{\partial}{\partial k_{\nu}}\left(q \oplus_{\ell}^{[p]} k\right)_{\lambda}\right|_{q=k=p}
$$

The the derivatives in the above equation give:

$$
\begin{aligned}
\frac{\partial}{\partial q_{\mu}} \frac{\partial}{\partial k_{\nu}}\left(q \oplus_{\ell}^{[p]} k\right)_{\lambda}= & -\tilde{\Gamma}_{\lambda}^{\mu \nu}{ }_{\lambda}+\frac{1}{2} \tilde{\Gamma}_{\lambda \beta}^{\alpha \beta} \tilde{\Gamma}_{\beta}^{\gamma \nu}{ }_{\beta}\left(\delta_{\alpha}^{\mu} q_{\gamma}+q_{\alpha} \delta_{\gamma}^{\mu}-\delta_{\alpha}^{\mu} p_{\gamma}-p_{\alpha} \delta_{\gamma}^{\mu}\right)+\frac{1}{2} \tilde{\Gamma}_{\lambda}^{\alpha \beta}{ }_{\lambda} \tilde{\Gamma}^{\mu \delta}{ }_{\alpha}\left(\delta_{\delta}^{\nu} k_{\beta}+k_{\delta} \delta_{\beta}^{\nu}-\delta_{\delta}^{\nu} p_{\beta}-p_{\delta} \delta_{\beta}^{\nu}\right) \\
& -\frac{1}{2} \partial^{\theta} \tilde{\Gamma}_{\lambda}^{\alpha \beta}{ }_{\lambda}\left(\left(\delta_{\alpha}^{\mu} q_{\theta}+q_{\alpha} \delta_{\theta}^{\mu}\right) \delta_{\beta}^{\nu}+\left(\delta_{\theta}^{\nu} k_{\beta}+k_{\theta} \delta_{\beta}^{\nu}\right) \delta_{\alpha}^{\mu}-\delta_{\theta}^{\mu} p_{\alpha} \delta_{\beta}^{\nu}-\delta_{\theta}^{\nu} \delta_{\alpha}^{\mu} p_{\beta}\right)
\end{aligned}
$$

so that:

$$
-\left.\frac{\partial}{\partial q_{\mu}} \frac{\partial}{\partial k_{\nu}}\left(q \oplus_{\ell}^{[p]} k\right)_{\lambda}\right|_{q=k=p}=\tilde{\Gamma}_{\lambda}^{\mu \nu}+\partial^{\theta} \tilde{\Gamma}_{\lambda}^{\mu \nu} p_{\theta}=\Gamma_{\lambda}^{\mu \nu}(p)
$$

[1] Max Born, Proc. R. Soc. Lond. A165 (1938) 291.

[2] Yu.A.GolÕfand, Soviet Physics JETP 10 (1960) 356. 
[3] S. Majid, Lect. Notes Phys. 541 (2000) 227 hep-th/0006166].

[4] G. Amelino-Camelia, Int. J. Mod. Phys. D 11 (2002) 35 gr-qc/0012051, G. Amelino-Camelia, Phys. Lett. B 510 (2001) 255 hep-th/0012238].

[5] J. Kowalski-Glikman, Phys. Lett. B 547 (2002) 291 hep-th/0207279.

[6] F. Girelli and E. R. Livine, Braz. J. Phys. 35 (2005) 432 gr-qc/0412079.

[7] D. Raetzel, S. Rivera and F. P. Schuller, Phys. Rev. D 83 (2011) 044047 arXiv:1010.1369 [hep-th]].

[8] L. N. Chang, Z. Lewis, D. Minic and T. Takeuchi, Adv. High Energy Phys. 2011 (2011) 493514 arXiv:1106.0068 [hep-th]].

[9] G. Amelino-Camelia, L. Freidel, J. Kowalski-Glikman and L. Smolin, Phys. Rev. D 84 (2011) 084010 arXiv:1101.0931 [hep-th]].

[10] G. Amelino-Camelia, L. Freidel, J. Kowalski-Glikman and L. Smolin, Gen. Rel. Grav. 43 (2011) 2547 [Int. J. Mod. Phys. D 20 (2011) 2867] arXiv:1106.0313 [hep-th]].

[11] G. Amelino-Camelia and S. Majid, Int. J. Mod. Phys. A 15 (2000) 4301 hep-th/9907110.

[12] C. Rovelli, Living Rev. Rel. 11 (2008) 5.

[13] L. Smolin, Gen. Rel. Grav. 43 (2011) 3671 arXiv:1004.0664 [gr-qc]l.

[14] H. -J. Matschull and M. Welling, Class. Quant. Grav. 15 (1998) 2981 gr-qc/9708054.

[15] H. -J. Matschull, Class. Quant. Grav. 18 (2001) 3497 gr-qc/0103084.

[16] L. Freidel and E. R. Livine, Class. Quant. Grav. 23 (2006) $2021 \mid$ hep-th/0502106.

[17] G. Amelino-Camelia, M. Arzano, S. Bianco and R. J. Buonocore, Class. Quant. Grav. 30 (2013) 065012 arXiv:1210.7834 [hep-th]].

[18] G. Amelino-Camelia, Phys. Rev. D 85 (2012) 084034 arXiv:1110.5081 [hep-th]].

[19] J. M. Carmona, J. L. Cortes and F. Mercati, Phys. Rev. D 86 (2012) 084032 [arXiv:1206.5961 [hep-th]].

[20] J. Kowalski-Glikman, Phys. Lett. A 286 (2001) 391 hep-th/0102098.

[21] J. Magueijo and L. Smolin, Phys. Rev. D 67 (2003) 044017 gr-qc/0207085].

[22] G. Amelino-Camelia, Nature 418 (2002) 34 [gr-qc/0207049].

[23] J. Magueijo and L. Smolin, Class. Quant. Grav. 21 (2004) 1725 gr-qc/0305055].

[24] J. Kowalski-Glikman, Lect. Notes Phys. 669 (2005) 131 hep-th/0405273.

[25] G. Amelino-Camelia, Symmetry 2 (2010) 230 arXiv:1003.3942 [gr-qc]].

[26] F. Mercati, talk given at Perimeter Institute (available online at http://pirsa.org/11110138/)

[27] G. Amelino-Camelia, Symmetry 4 (2012) 344 arXiv:1111.5643 [hep-ph]|.

[28] G. Gubitosi and F. Mercati, Class. Quant. Grav. 30 (2013) 145002 arXiv:1106.5710 [gr-qc]].

[29] G. Amelino-Camelia, M. Arzano, J. Kowalski-Glikman, G. Rosati and G. Trevisan, Class. Quant. Grav. 29 (2012) 075007 arXiv:1107.1724 [hep-th]].

[30] L. -Q. Chen, arXiv:1212.5233 [gr-qc].

[31] S. Majid and H. Ruegg, Phys. Lett. B 334 (1994) 348 hep-th/9405107.

[32] J. Lukierski, H. Ruegg and W. J. Zakrzewski, Annals Phys. 243 (1995) 90 hep-th/9312153.

[33] G. Amelino-Camelia, Phys. Lett. B 392 (1997) 283 [gr-qc/9611016.

[34] G. Amelino-Camelia and S. Majid, Int. J. Mod. Phys. A 15 (2000) 4301 hep-th/9907110].

[35] L. Freidel and E. R. Livine, Phys. Rev. Lett. 96 (2006) 221301 hep-th/0512113.

[36] L. Freidel and L. Smolin, arXiv:1103.5626 [hep-th].

[37] A. Banburski, arXiv:1305.7289 |gr-qc|. 